EUROPEAN UNIVERSITY INSTITUTE DEPARTMENT OF ECONOMICS

EUI Working Paper ECO No. 2002/24

Dating the Euro Area Business Cycle

\author{
Michael Artis, Massimiliano Marcellino \\ and \\ Tommaso Proietti
}

BADIA FIESOLANA, SAN DOMENICO (FI) 
All rights reserved.

No part of this paper may be reproduced in any form without permission of the author(s).

(C)2002 Michael Artis, Massimiliano Marcellino and Tommaso Proietti

Published in Italy in November 2002

European University Institute

Badia Fiesolana

I-50016 San Domenico (FI)

Italy 


\title{
Dating the Euro Area Business Cycle
}

\author{
Michael Artis \\ Massimiliano Marcellino \\ European University Institute and CEPR Università Bocconi, Igier and CEPR \\ Tommaso Proietti \\ Università di Udine and European University Institute
}

\begin{abstract}
In this paper we compare alternative approaches for dating the Euro area business cycle and analyzing its characteristics. First, we extend a commonly used dating procedure to allow for length, size and amplitude restrictions, and to compute the probability of a phase change. Second, we apply the modified algorithm for dating both the classical Euro area cycle and the deviation cycle, where the latter is obtained by a variety of methods, including a modified HP filter that reproduces the features of the BK filter but avoids end-point problems, and a production function based approach. Third, we repeat the dating exercise for the main Euro area countries, evaluate the degree of syncronization, and compare the results with the UK and the US. Fourth, we construct indices of business cycle diffusion, and assess how spread are cyclical movements throughout the economy. Finally, we repeat the dating exercise using monthly industrial production data, to evaluate whether the higher sampling frequency can compensate the higher variability of the series and produce a more accurate dating.
\end{abstract}

JEL Classification: E32, E39

Keywords: Business cycle, Euro area, cycle dating, cycle synchronization 


\section{Introduction}

The business cycle can be defined as a broadly-based movement of economic variables in a sequentially oscillatory manner. The term 'cycle' is a misnomer to the extent to which it suggests a regular periodicity; one of its features is that the length and depth (duration and amplitude) of the cycle seems to vary. Indeed one of the current preoccupations of US business cycle experts (e.g., Stock and Watson 2002) is to explain the apparent lengthening of the cycle there in recent history.

There are several other reasons for taking an interest in the cycle. The evolution of the cycle carries with it an evolution in variables of considerable consequence for policy-makers: indeed, policy-makers are commonly depicted as endeavouring to reduce the extent of fluctuations by exercising stabilization policy. A closely related interest has been in the use of business cycle evidence in the context of optimal currency area theory and its indication for the optimality of monetary union. Other things equal, business cycle symmetry is a positive indicator for monetary union as it indicates that a single monetary policy will be broadly appropriate for all participants in the monetary union. On the other side, an asymmetry of business cycle experience is usually treated as a negative indicator for participation in monetary union. Now is a good time to begin the study of the cyclical properties of the Eurozone economy, as it is part of the assembly of facts about the "new" economy given life by the commitment to a single monetary policy.

The literature recognizes two broad definitions of the cycle, the so-called classical cycle and the growth or deviation cycle. The difference between the two is conceptually simple: in the case of the deviation cycle, turning points are defined with respect to deviations of the rate of growth of GDP from an appropriately defined trend rate of growth. There is a large technical literature which is concerned with the best method of extracting a trend from the data, and it turns out that the method adopted may carry quite important implications for the subsequent dating of the turning points. The classical cycle, by contrast, selects its tuning points on the basis of an absolute decline (or rise) in the value of GDP.

In early post-war decades, especially in Western Europe, growth was relatively persistent and absolute declines in output were comparatively rare; the growth cycle then seemed to be of more analytical value especially as inflexions in the rate of growth of output could reasonably be related to fluctuations in the levels of employment and unemployment. In more recent decades, however, 
there have been a number of instances of absolute decline in output, and popular description at any rate has focussed more on the classical cycle (for example there is a widespread impression that a recession defines itself as two consecutive quarters of absolute decline). In addition, the concern mentioned above that de-trending methods can affect the information content of the series in unwanted ways, has reinforced the case for examining the classical cycle.

In this paper we analyze the business cycle in the Eurozone and in its main constituent economies, comparing both concepts of the cycle. Previous work documenting the cyclical experience of the Eurozone economy is quite sparse, being limited essentially to the paper by Agresti and Mojon (2001), which applies the notion of a growth or deviation cycle based on the use of the bandpass filter, and work by Harding and Pagan (Harding and Pagan, 2001, Pagan, 2002) which focuses also on the notion of the classical cycle. We adopt Pagan's dating methodology, but extend it to compute the probability of phase switch, introduce depth or amplitude restrictions, and dating monthly series. Our results on dating the cycle largely confirm the findings in these previous studies, with some differences we note below.

We employ GDP as a basic broad-based measure of economic activity, but also study the cyclical evolution of other variables, such as employment, investment, consumption and net exports to examine their possible role in relation to the cycle, rather than as giving indications for a different dating of the cycle itself.

Any study of the Eurozone economy faces a problem of data availability. The Eurozone only came into being on the 1st January 1999, and the study of business cycles needs a larger sample than three-and-a-half years. To extend the data back in time encounters the problem of aggregation when exchange rates are pone to change: in these circumstances there is no "perfect" method of aggregation. We have employed, for the most part, the data that have been constructed for the ECB's Area-wide model, conducting a check against the main alternatively-generated series, that produced by Beyer, Doornik and Hendry (2001) in two instances (the comparison allows us to conclude that our results are relatively robust to the method of aggregation).

In section 2 we present our analysis of the Eurozone broad aggregates. Dating of the classical cycle is accomplished by employing our modified Harding and Pagan's algorithm. For the deviation cycle we consider the most popular methods of detrending, those due to Hodrick and Prescott 
(1997, HP) and to Baxter and King (1999, BK), going on to present estimates which rely either on a technique suggested by Harvey and Trimbur (2002)or on a modified HP filter that reproduces the features of the BK filter but avoids end-point problems. In addition we also experiment with a definition of the trend based on a production function approach of the type that is used in calculating output gaps.

In section 3 we repeat the estimation for the main Eurozone countries (France, Germany, Italy), for which we can obtain consistent output series, together with the UK. We compare timing and other measures of the cycle, in addition including the US in the comparison.

Section 4 seeks to employ higher frequency data (the GDP data are quarterly), namely (monthly) industrial production data. The advantage of concentrating on this series is that its higher frequency should enable a more precise dating of the cycle whilst it is already known that the most cyclically-sensitive component of GDP is in fact industrial production. The disadvantages are that industrial production is a small proportion of total output in many European economies, and has been declining over the period as a whole. Moreover, its higher variability requires a careful use of the constrained dating algorithm.

Section 5 constructs indices of business cycle diffusion, and applies econometric techniques aimed at assessing how spread are cyclical movements throughout the economy. Section 6 exploits the diffusion indices to evaluate the degree of convergence across the Euro area countries. Section 7 summarizes and concludes. The paper contains a number of appendices which lay out the technical detail of what has been done.

\section{The aggregate cycle}

This sections analyses aggregate time series data available for the Eurozone both from the classical and deviation cycle perspective. The emphasis is on the Euro area GDP, but we also consider its decomposition into expenditure components and the labour market. The national accounts aggregates are measured at constant prices. 


\subsection{Classical BC}

Classical cycle dating commonly follows the algorithm developed by Bry and Boschan (1971), the so-called BB algorithm, which is designed in its original form for monthly data and claims to replicate the NBER procedures for a single series (this would commonly be that of industrial production at a monthly frequency). More recently, the algorithm has been adapted to the quarterly frequency (by e.g., Harding and Pagan, 2001, in which the resultant algorithm is dubbed the BBQ algorithm, and McDermott and Scott, 1999).

This is the lead that we follow here. The classical cycle makes relatively few strict definitional demands: the traditional ones are that a peak (trough) is identified as preceding an absolute fall (rise) in the chosen measure of economic activity, and that peaks and troughs should alternate. Such a sparse definition might admit blips and bumps in economic activity which could not be recognised as cycles and it is normal to add minimum duration requirements for each phase of the cycle and for the cycle as a whole, as here where cyclical phases must exceed two quarters to be recognized as such and where the cycle as a whole must last at least five quarters. The algorithm is explained in more detail in Appendix A, where we show that, using the theory of Markov chains, it is relatively straightforward to implement minimum duration requirements, alternation of turning points, and to taylor the dating algorithm for specific needs.

Our classical business cycle chronology is presented compactly in Figure 1. Two alternative measures of Eurozone GDP are employed: the "AWM series" (this is the data set underlying the application of the "Area-wide model" (Fagan, Henry and Mestre, 2001) and the "BDH" series, constructed by Beyer, Dornik and Hendry (2001) . The former has a longer sample period (19702001) than the latter (1980-2001) and thus reveals one more cycle. Otherwise, the three cycles identified in the shorter data period overlap almost exactly, the only difference being in the location of the last trough which is anticipated by one quarter if one takes the BDH measure, and the three decades from 1970 comprise four cycles altogether. The chronology of turning points, not surprisingly, is also exactly as in Harding and Pagan (2001). It should also be noticed that the two quarters recession in 1982 is a minor event and would be censored if the dating algorithm was tailored to impose ties on the depth of recessions and expansions; we will return to this issue shortly. 
Figure 1 also presents the expansion/recession classification based on GDP growth rates, which basically confirms the previous findings. More details on the relationships among classical, deviation and growth cycles are provided in Appendix B. ${ }^{1}$

Table 1 displays some descriptive statistics. For the moment we concentrate on those pertaining to GDP (denoted YER in the table). There is a notable asymmetry between the average length of expansions and recessions, the former much longer (28 quarters) than the latter (3 quarters), which is to be expected of classical cycles in a growing economy. The probabilities of being in one or other phase reflect the relative values of these phase lengths (about 90\% versus 10\%). The amplitudes of the expansion periods are also much bigger than those of the recession periods. "Steepness", following the suggestion of Harding and Pagan (2001), is measured as the quotient of the amplitude and the duration of the phase. Expansions last longer, and are steeper than recessions, which are quite brief and yet more gently sloped.

Table 1 also displays comparable information for a number of other series - notably the national accounts categories pertaining to private and government consumption (PCR and GCR), fixed capital formation (ITR), imports and exports of goods and services, and net exports (MTR, XTR, Net Imp) and inventory change (SCR), together with employment (LNN), productivity (LPROD), unemployment (URX) and unit labour costs (ULC). Standard theory would suggest that investment and inventories are likely to be the most cyclical components of GDP, and this expectation is borne out in the data: more cycles are identified, the recession and expansion probabilities are more nearly equal and the steepness of the phases is more nearly equal. It is not surprising perhaps to find, on the other hand, that the cyclical behaviour of private consumption is much in line with that of GDP as a whole, whilst government consumption is the smoothest component of all. Exports and imports of goods and services, and even more, the net of the two, seem to be highly cyclical in their behaviour.

\footnotetext{
${ }^{1}$ In the comparison of Appendix B we also include the Eurocoin indicator, constructed by Altissimo et al. (2001), and regularly published by the CEPR at http://www.cepr.org/data/eurocoin/.
} 


\subsection{Deviation Cycles}

An alternative business cycle definition refers the recurrent, though not strictly periodic, deviations around the long term path of the series. The deviation or growth cycle typically represents an unobserved component and various methods and filters have been proposed to extract it, both in the model-based and the nonparametric frameworks. Appendices D, E and F review briefly the univariate methods that will be employed, among which the Baxter and King (1999) filter, a bandpass version of the Hodrick and Prescott (1997) filter and the Harvey and Trimbur (2002) cyclical filters derived within an unobserved components model. In the sequel we shall denote the deviation cycle by $\psi_{t}$.

Figure 2 presents several measures of the deviation cycle in the Euro area GDP, with the associated turning points detected by the dating algorithm BBQDC2 of appendix A.4, with restrictions on the size of the fluctuations that will be discussed shortly. The first measure is the Baxter and King cycle, that is available for the central part of the sample excluding the first and last 12 quarters; the second, displayed on the upper right panel, is the HP bandpass filtered cycle, which results by subtracting the HP trend with smoothing parameter $\lambda=0.52$, which defines a lowpass filter dampening the fluctuations with a period smaller than 5 quarters (1.25 years, e.g. high frequency noise) from the HP trend with smoothing parameter $\lambda=677$, which in turn defines a lowpass filter cutting off the fluctuations with a period smaller than 8 years; later on we shall refer to these filters respectively as $\mathrm{HP}(1.25)$ and $\mathrm{HP}(8)$, and in general $\mathrm{HP}(\cdot)$ will denote a lowpass filter with cut-off period in years indicated in parenthesis. The bandpass filter stemming from the subtraction extracts the fluctuations with a period between 5 quarters and 8 years, and in this respect produces estimates of the cycle that are comparable, although slightly noisier to the BK cycle, without suffering from unavailability of the end of sample estimates.

The bottom panels display measures of the output gap derived respectively from a bivariate model of GDP and CPI inflation and a multivariate model based on total factor productivity, labour force participation rates, the unemployment rate, capacity utilisation and CPI inflation, implementing the production function approach, see Proietti, Musso and Westermann (2002) for details. ${ }^{2}$ The

\footnotetext{
${ }^{2}$ The PFA model considered here is the one featuring pseudo-integrated cycles. The paper highlights the uncertainty issue arising from model-based univariate estimation of the output gap, and performs a rolling forecast exercise whose
} 
first equation of the bivariate model decomposes output into potential, $\mu_{t}$, represented as a local linear trend, and the output gap, $\psi_{t}$, a stationary $\operatorname{ARMA}(2,1)$ process:

$$
\begin{array}{lll}
y_{t} & =\mu_{t}+\psi_{t}, & \\
& & \\
\mu_{t}=\mu_{t-1}+\beta_{t-1}+\eta_{t}, & \eta_{t} \sim \operatorname{NID}\left(0, \sigma_{\eta}^{2}\right) \\
\beta_{t}=\beta_{t-1}+\zeta_{t}, & \zeta_{t} \sim \operatorname{NID}\left(0, \sigma_{\zeta}^{2}\right) \\
\psi_{t}=\rho \cos \lambda_{c} \psi_{t-1}+\rho \sin \lambda_{c} \psi_{t-1}^{*}+\kappa_{t}, & \kappa_{t} \sim \operatorname{NID}\left(0, \sigma_{\kappa}^{2}\right) \\
\psi_{t}^{*}=-\rho \sin \lambda_{c} \psi_{t-1}+\rho \cos \lambda_{c} \psi_{t-1}^{*}+\kappa_{t}^{*}, & \kappa_{t}^{*} \sim \operatorname{NID}\left(0, \sigma_{\kappa}^{2}\right)
\end{array}
$$

where $\eta_{t}, \zeta_{t}, \kappa_{t}$, and $\kappa_{t}^{*}$ are mutually independent. The price equation is a version of Gordon's triangle model of inflation, specified as follows:

$$
\begin{aligned}
& p_{t}=\tau_{t}+\sum_{k} \delta_{k}(L) x_{k t} \\
& \tau_{t}=\tau_{t-1}+\pi_{t-1}^{*}+\eta_{\pi t} \quad \eta_{\pi t} \sim \operatorname{NID}\left(0, \sigma_{\eta \pi}^{2}\right), \\
& \pi_{t}^{*}=\pi_{t-1}^{*}+\theta_{\pi}(L) \psi_{t}+\zeta_{\pi t} \quad \zeta_{\pi t} \sim \operatorname{NID}\left(0, \sigma_{\zeta \pi}^{2}\right) \text {. }
\end{aligned}
$$

where the regressors are commodity prices $x_{k t}$ and the nominal effective exchange rate of the Euro. The only link between the prices and output equations is the presence of $\psi_{t}$ as a determinant of underlying inflation, $\pi_{t}^{*}$. The reduced form is

$$
\Delta^{2} p_{t}=\theta_{\pi}(L) \psi_{t-1}+\sum_{k} \delta_{k}(L) \Delta^{2} x_{k t}+(1+\vartheta L) \epsilon_{t}
$$

so that $\theta_{\pi}(1)=0$ (the level effect is zero) the output gap has only transitory (change) effects on inflation.

The notion of an output gap is more specialised than the deviation cycle in output, since it provides a measure of inflationary pressures. This poses a new issue to the dating of the gaps: Pagan proposes to score $\psi_{t}>0$ and $\psi_{t}<0$, as the interest lies in dating periods in which the inflationary pressures are positive or negative. However, as the evidence reported in Proietti, Musso and Westermann (2002) clearly points out, it is the change effect associated to $\Delta \psi_{t}$ that is more relevant than the level effect exerted by the output gap, which brings us back to the problem outcome is that the bivariate model produces the best forecasting performance in the test period considered. 
of dating expansions and recessions in the level of $\psi_{t}$. We also notice in passing that the scoring of the gap according to whether it is positive or negative is a by-product of BBQDC2.

Figure 2 shows a broad agreement in the identified turning points: the 74.1 and 80.1 peaks are common to the four representations. The location of the start of the $90 \mathrm{~s}$ recession is more uncertain since there are two neighbouring local maxima at the beginning of 1990 and 1992, which is featured by the expenditure components and the GDP of individual countries. Also the beginning of the 80 s expansion is scored differently by the different methods.

As stated above, the BBQDC2 dating algorithm featured restrictions on the amplitude of the fluctuations: in its first stage, by which change of signs in $\psi_{t}$ are identified by using the usual BBQ dating rules on the cumulated cycle, we amended the definition of the expansions and terminating sequences as in appendix A.6 by setting $c=0.005$, which amounts to censor the fluctuations around zero with amplitude less than $0.5 \%$ of total GDP. Amplitude restrictions are perfectly sensible, although they inevitably underlie some degree of arbitrariness, as the amplitude of the deviation cycle differs according to the signal extraction model or technique, see eg. figure 2, the maximum amplitude usually being achieved by linear detrending of the series. Nevertheless they enhance one of the three key features (the three "D"s) that are used to characterise economic fluctuations as business cycle fluctuations: duration is ensured by the ties imposed by the dating algorithm: complying with the BBQ convention, we impose a minimum duration of 5 quarters for a full cycle and 2 quarters for a phase. The second characteristic of the business cycle, diffusion, is automatically enforced when we deal with an overall measure of economic activity, such as GDP. The third, depth, needs to be enforced setting up additional restrictions as in appendix A.6, these rules are usually judgemental, but they can be drawn from the history of the series under investigation.

Table 2 presents some characteristics of the deviation cycles extracted by the HP quarterly bandpass filter (appendix E.1) not using any censoring rule on the amplitude of the fluctuations. This results in a relatively large number of turning points, compare e.g. the YER series with figure 2, and affects the duration and the amplitude statistics. The stylised fact that is however robust to the choice of censoring rules is that the average amplitude of recessions and expansions is about the same, as implied by the symmetry of the cyclical model or signal extraction filter. It 
is important to stress that this is an implication of the representation of the cycle that is chosen, although a model based framework allows to test for business cycle nonlinearity and asymmetry (see, e.g.. Proietti, 1998). Table 2 confirms that investment (ITR) is one of the most cyclically variable expenditure component of GDP, featuring an average amplitude of 5\% for both phases. Employment and unemployment are now less cyclical than GDP.

The Harvey and Trimbur (2002) cycles, see Appendix F, estimated by the Kalman filter and smoother using a second order representation for the trend and the cycle, are reported in figure 3 for a few selected series, along with recession probabilities and the probabilities of peaks and troughs (on a reverse scale). The estimated parameters imply a smooth representation for the cycle, $(1-\phi L)^{2} \psi_{t}=\kappa_{t}$, i.e. a second order autoregressive representation with two common real roots at the zero frequency. As a result the estimated component is relatively smooth and this has a bearing on turning points characterisation which need not to be sharp (the ITR series being an exception). The chronology for GDP arising from this representation is slightly different from that obtained from the other approaches that we considered. Fundamentally, the depth of the downturn at the beginning of the 80ies and the subsequent recovery are emphasised; this is a consequence of the smoothness prior imposed on the cyclical dynamics, which makes the extracted component much more stable and less responsive to the kind of shorter run fluctuations that occurred in the mid seventies and at the end of the nineties. For instance, the estimated cycle for ITR does not fall below zero in 1975 and no trough is detected. We also notice that the recession probabilities are never sharp, which is again an expression of the fundamental trade-off between smoothness in the signal evolution and the resolution, or sharpness, in detecting cyclical changes.

\section{Country specific cycles}

Our analysis of country specific cycles focuses on two data sets, the first relating to the GDP at constant prices for five countries, Germany, France, Italy, UK and the USA, starting from 1970 and available from various sources, among which the OECD Main economic indicators and the US Bureau of Economic Analysis. For Germany the series, made available by the IFO, has been seasonally adjusted, corrected for working days and the level shift due to reunification, using the basic structural model with regression effects. The Eurozone series is used for comparison. The 
second set is produced by Eurostat and provides a highly comparable set of statistics about real GDP based on the new system of national accounts (ESA95), but for a shorter sample.

\subsection{Classical Cycles}

Figure 4 presents the turning points of the classical BC for the Euro Area, Germany, France, Italy, UK and the USA, identified using the $\operatorname{HP}(1.25)$ filtered series on the the first data set. We recall that this is a low-pass filter dampening the fluctuations with a period less than five quarters, which strictly do not pertain to the business cycle.

We next address the issue of synchronisation and concordance among the country specific business cycles. The dating algorithm, applied to the HP(1.25) filtered GDP series furnishes the indicator variables of the state of the economy, $\mathrm{R}_{t}$ and $\mathrm{E}_{t}$ (recession and expansion, respectively). The index of concordance between the classical BC for the individual countries and the Euro Area aggregate cycle, $I_{i j}$, is simply the percentage of time units spent in the same phase, also known as the simple matching similarity coefficient. The mean corrected concordance index, is $I_{i j}^{*}=I_{i j}-\bar{I}_{i j}$, where $\bar{I}_{i j}$ is the estimate of the expected value of the index under the assumption of independence, which represents the fraction to be expected if there were no relationship between the cycle in the two countries. Finally, dividing $I_{i j}^{*}$ by its asymptotic standard error estimated nonparametrically using a Newey-West estimator, we get the standardized index, which is reported in Table 3 and can be interpreted as a t-statistic for the null hypothesis of independence of the cycles (see Appendix C for details).

Looking at the Euro area as a whole, the concordance is lowest with the UK, though still statistically different from zero, highest with the countries within the Euro area, as expected, and intermediate with the US. Germany, France and Italy are also the group of countries with the highest cross concordances. The highest concordance for the UK is with the US.

Harding and Pagan (2001, 2002, Pagan, 2002) also propose to regress the recession indicator for one country on the same indicator for another country, and evaluate BC independence using the t-statistic for the significance of the parameters, computed using HAC standard errors. The results, reported in table 4 now suggest that the UK cycle is even independent of that of the EA, Germany and France, whereas there is a significant association with Italy and the US and cross 
independence across the Euro area countries is strongly rejected.

The analysis of the Eurostat series (see figure 5), available for a shorter time span, beginning in 1980 for most countries, is useful to pinpoint an additional peak that was not identified from the other Eurozone series considered before, taking place in the second quarter of 2001. This is mainly due to Germany, but is also anticipated in the series for Finland, Belgium, the Netherlands and Austria. Figure 6 illustrates that when high frequency dynamics, intended as those fluctuations with periodicity less than 5 quarters, are filtered out by a low-pass HP filter, fewer turning points are found.

\subsection{Deviation Cycles}

Figure 7 plots the deviation cycles, and the squared coherence between the Euro area deviation cycle and that of the countries belonging to the first data set. The plot suggests lower coherence with the US and UK cycles, confiriming the previous finding

To investigate further the issues of concordance and synchronisation we also report the standardised concordance index (table 5) and the robust test for cycle independence (table 6). The results largely confirm the previous outcome: there is a high degree of sinchronisation within the Euro area, with the lowest concordance for the US and intermediate for the UK, and in all cases the hypothesis of business cycle independence is rejected.

\section{Monthly indicators}

This section focuses on the analysis of business cycles in monthly industrial production series for most European countries and the US. The series, seasonally adjusted, are drawn from the OECD Main Economic Indicators and cover a sample period that differs for the individual countries, but is usually rather long. We set off by identifying the major additive outliers; the strategy was to add pulse intervention variables one at a time corresponding to the sample observation that had a standardised auxiliary residual for the irregular component of the local linear trend model greater than 4 in absolute value. See Harvey and Koopman (1992) for the definition of auxiliary residuals and their use for outlier and structural break detection. 
Despite the outlier correction, too many turning points are identified due to the presence of high frequency noise that may result from intrinsic volatility, underadjustment of working days variation and other events such as moving festivals and strikes. This problem may be tackled either by setting up amplitude restrictions or by smoothing the series. Figures 8 and 9 illustrate the impact of smoothing by different filtes on dating the classical business cycle. The first plot refers to $\mathrm{HP}(1.25)$, dampening the fluctuations with a period less than 15 months, whereas the second is based on $\mathrm{HP}(4)$, and thus on a smoother signal - compare with the use of different moving averages in the Bry and Boschan routine. We employ the simulation smoother to estimate the probability of recessions and of a turning point. $\mathrm{HP}(1.25)$ produces a relatively large number of cycles. However, some stylised facts are common. The turning points characterisation (those of a trough are plotted on a reversed scale) is not particularly sharp as they have attached a rather low probability, which is smeared on adjacent data points, but the recession and expansion probabilities are rather sharp. This results because of the uncertainty surrounding the signal estimates in an environment where noise contamination is at relatively high levels. The shape of the recession probabilities highlights some interesting features, among which it is remarkable that expansion and recession probabilities behave asymmetrically (expansion termination is usually quicker than recession termination). When average growth is reduced, as it occurs for France in the second half of the 70s, the probability of recession is higher. For Germany, France and Italy we find a high degree of synchronisation.

As for concordance analysis, Table 11 reports the standardized concordance index for all available countries. These are in general all rather high for the countries in the Euro area, whose business cycles turn out to be also not independent from those of the UK and US. Similar results are obtained for the HP- deviation cycles, see Table 12.

\section{Diffusion and Multivariate Business Cycle Assessment}

An index of business cycle diffusion measures the percentage of economic time series in a certain state, e.g. recession. It typically aims at assessing on a $0-1$ continuous scale how spread are business cycle movements throughout the economy, by looking at several phenomena that have known nature, eg. coincident or leading. 
There are two ways in which diffusion indexes can be constructed. The first amounts to score each individual time series and then take the cross-sectional average

$$
D_{t}=\frac{1}{N} \sum_{i=1}^{N} S_{i t}, \quad t=1, \ldots, T
$$

where $S_{i t}$ takes value 1 in recessions and zero otherwise, and $N$ is the cross-sectional dimension.

It can be worth weighting the series considered according to their economic relevance and/or their proved efficacy in signalling recessionary events. If a system of (possibly time-varying) weights $w_{i t}$ is available then

$$
D_{t}=\sum_{i=1}^{N} w_{i t} S_{i t}, \quad t=1, \ldots, T, \quad \sum_{i} w_{i t}=1 .
$$

The underlying model is that the aggregate index, $D_{t}$, is a finite mixture of two states Markov processes, the mixture probabilities being given by $w_{i t}$. Suppose that the individual time series are the components of an aggregate $y_{t}=\sum_{i} w_{i} y_{i t}$ and that we score recessions according to the calculus rule, that is $S_{t}=\mathrm{I}\left(\Delta y_{t}<0\right)$, where $\mathrm{I}(\cdot)$ is the indicator function, then

$$
E\left(S_{t}\right)=P\left(\sum_{i} w_{i} \Delta y_{i t}<0\right)>E\left(D_{t}\right)=\sum_{i=1}^{N} w_{i t} E\left(S_{i t}\right),
$$

so that the diffusion index does not measure the probability of a recession in the aggregate series; rather it measures the proportion of the aggregate that is in a recession.

The second method to compute diffusion indexes exploits the dating algorithm of Appendix A, where the transition probabilities are computed using the probability attached to expansion and recession terminating sequences (ETS and RTS, respectively) in the following way:

$$
\mathcal{P}_{t}^{(E T S)}=\sum_{i=1}^{N} w_{i t} \mathrm{I}\left(\mathrm{ETS}_{i t}\right), \quad \mathcal{P}_{t}^{(R T S)}=\sum_{i=1}^{N} w_{i t} \mathrm{I}\left(\mathrm{RTS}_{i t}\right) .
$$

An expansion terminating sequence defines a candidate point for a peak, e.g. in the quarterly case, expansion is terminated at time $t$ when both $\left(\Delta y_{t+1}<0\right)$ and $\left(\Delta_{2} y_{t+2}<0\right)$ occur. On the other hand, a recessionary pattern is concluded by a trough at time $t$ if both $\left(\Delta y_{t+1}>0\right)$ and $\left(\Delta_{2} y_{t+2}>0\right)$ take place. Under the stated rule, the transition probabilities depend on the sum of the weights of the series that are in those two terminating sequences. Again, the underlying assumption is that the aggregate $\mathrm{ETS}_{t}$ is a finite mixture of cross-sectional ETS $i t$ and the dating 
algorithm furnishes probabilities that must be interpreted as $P\left(D_{t}=1\right)$, not as $P\left(S_{t}=1\right)$, and loosely speaking are a smoothed version of the previous diffusion index.

Assessing the diffusion of the business cycle in the Euro area requires the evaluation of sector and country specific data, and many disaggregated time series, but given our data availability for the present being we consider three sets of data that can be used to produce a multivariate assessment of the classical cycle in the Euro area: the first is made up of the 5 expenditure components of GDP (private consumption, government consumption, fixed capital formation, net exports and variation in stocks) considered in section 2.1 . The set of weights is immediately available as the GDP shares. The second set considers total factor productivity, as measured by the Solow's residual using the time averaged labour share $\alpha=0.35$ and a constant returns to scale Cobb-Douglas technology, total employment, and capital. This yields another decomposition of log output The weights are proportional to the Cobb-Douglas weights. The third set consists of the 12 industrial production series for the Euro area countries; the weights were obtained from the total Gross Value added at basic prices for the year 2001, available from the individual countries account (except for Greece, Luxembourg and Ireland, for which it was interpolated from total GDP estimates).

Figure 10 presents the diffusion indexes emerging from the three sets. The plot reveals the following: the diffusion of recessions is higher for industrial production and there is a tendency to peaking with a short lead, usually one quarter. A recessionary pattern that is idiosyncratic to the industrial sector can be found in 1987. Industrial production and the variables in the production function approach signal entry to a recessionary state in 1990 and 1991 respectively, whereas the GDP by expenditure diffusion peaks in 1992. For the latter, an important contribution is made by labour which peaks before GDP, as noticed before. The diffusion indexes behave asymmetrically along the time axis, this feature stems from the fact that the proportion of time series entering a recession is larger than that leaving it, which explains the positively skewed pattern.

The example also illustrates that weighting is a crucial issue: if we were to combine the three diffusion indexes into an aggregate one by simple averaging, then we would presumably overstate the diffusion, due to the influence of the IP diffusion index that dominates the others. 


\section{Degree of convergence within the Euro Area}

If a diffusion index is constructed from country specific business cycles, as we did for industrial production, it can be argued that $D_{t}$ provides at least some information on the degree of convergence: $D_{t}\left(1-D_{t}\right)$ is a measure of dispersion and it is a maximum when $D_{t}=0.5$, whereas it gets to zero when all the series are in the same state. In this perspective, figure 10 highlights that there is no tendency to cluster around the extremes ( 0 or 1$)$ as time progresses, especially compared to the 70 s and the 80 s.

There appears to be no systematic tendency for convergence or divergence in the classical business cycle sense. Judging from the cross-sectional dispersion of the member countries growth rates around the Euro area average (constructed with fixed weights), it is possible to establish that dispersion peaked in the seventies, declined until around 1985, it is relatively small during 1985-1990 and 1995-1998 and rises again in 1990-1995 and 1999-2000. Basically, it increases in recessionary periods, but the long term trend in the volatility of growth is that in the last 15 years it is lower than in the period 1970-1985. A reduction in volatility implies, cœteris paribus, a smaller frequency of recessionary events in a given time span. However, the average growth rates characterising several of the 12 countries are reduced, not by the same amount, and this increases the frequency of country specific cycles in the classical sense. As a result, at the end of the sample we find less ample but more frequent recessionary patterns, but we have no decisive evidence for either the presence of convergence or divergence.

When we turn to the deviation cycle, we find stronger support for convergence, by which we mean a systematic tendency for cross-sectional dispersion to shrink over time. If the countries have already converged, we should not assist to any systematic tendency in dispersion to increase over time. Figure 11 displays the time pattern of the weighted variance measure:

$$
\frac{1}{\sum_{i} w_{i}} \sum_{i=1}^{N} w_{i}\left(\psi_{i t}-\bar{\psi}_{t}\right)^{2}, \quad \bar{\psi}_{t}=\frac{1}{\sum_{i} w_{i}} \psi_{i t},
$$

where $\psi_{i t}$ are the country specific deviation cycles extracted by the HP bandpass filter, with the circle representing the point estimates and the index plot is a two sided exponentially weighted 
moving average of those estimates. The alternative measure

$$
\sum_{i} \sum_{i<j} w_{i} w_{j}\left(\psi_{i t}-\psi_{j t}\right)^{2}
$$

based on individual contrasts, rather then on the deviation from the average cycle, produces similar results and is not presented.

From the plot there appears to be a downward tendency interrupted around the turning points. The plot of the individual cycles and the aggregate cycle illustrates that the dispersion is high around 1990 with Finland behaving more idiosyncratically. Luxembourg displays more ample fluctuations.

\section{Conclusions}

This paper has reported the results of technical exercises in the dating of the Eurozone business cycle and the cycles of the main constituent economies. We distinguished between the classical and deviation (or growth) cycle, and used what we regard as best-practice techniques to identify these cycles, in every case concentrating upon a single, univariate summary of economic activity, GDP or industrial production. A number of topics for further research are suggested by the identification we have made. One, which we have already pursued in this paper, is to examine the issue of synchronicity or coherence between the cycles. In future research one would expect to be able to track movements in the coherence of the cyclical experience of the Eurozone economies, whether in the direction of greater convergence or not. Other topics can easily be suggested: thus, following identification of the cycle, one would hope to be able to build leading indicators; and to be able to explain the main determinants of cyclical experience and its evolution over time. As we maintained in the introduction to the paper, this is a particularly good time to initiate further studies of the European business cycle experience, as the adoption of the Euro gives the Eurozone economy a new identity and can be expected to have important effects on the Eurozone cycle.

It may indeed be time to ponder on the following. In the United States the NBER's dating committee has established a business chronology over a long period of time which is widely regarded as the authoritative dating of the US cycle. Economists who come up with a new technique for business cycle identification "prove" their technique by comparing their results with the NBER 
chronology. The NBER's committee comprises economists with expertise in various sectors of the economy and its approach is avowedly a multivariate one: techniques such as those we deployed in this paper applied to a single series can be regarded as "office assistants", helpful in establishing the dating, not dictating it. It would be good for Europe to establish a comparably authoritative chronology for its business cycle, perhaps through similar means - perhaps through a different one. At risk of stating the obvious, it bears pointing out that the European situation is different in many ways from the one that faces analysts of the American cyclical experience. Not least, because of the short history of the Eurozone economy, country experts might be needed more than sectoral experts. Historical analysis would be complicated by national differences, but all the more necessary for this reason. A complaint that is often made against the NBER procedure is that it can take a long while, in real time, to establish a turning point, partly for reasons to do with data revisions and availability; some of those problems are more acute in the European setting. Then also, it might be argued that with techniques at hand today nothing more is really needed than reliable GDP estimates, or data from a factor analysis that proxy GDP, with the benefit of being robust to revision, to provide reliable dating of the cycle. To provide more accurate dating, better monthly data are certainly needed. 


\section{References}

Agresti, A. and Mojon, B. (2001), Some Stylised Facts on the Euro Area Business Cycle", ECB Working Paper, No. 95,

Altissimo, F., Bassanetti, A., Cristadoro, R., Forni, M., Hallin, M., Lippi, M., Reichlin, L. and Veronese, G. (2001), EuroCOIN: A Real Time Coincident Indicator of the Euro Area Business Cycle, CEPR Working paper No 3108.

Baxter M., and King R.G. (1999), "Measuring business cycles: approximate Band-pass filters for Economic Time Series, Review of Economics and Statistics, 81, 4, 575-93.

Beaudry, P., and Koop, G. (1993), “Do recessions permanently change output?”, Journal of Monetary Economics, 31, 149-163.

Beyer, A., Doornik, J., and Hendry, D.F. (2001), "Constructing Historical Euro-Zone Data", Economic Journal, 111, 308327.

Bry, G. and Boschan C. (1971), Cyclical Analysis of Time Series: Selected Procedures and Computer Programs, National Bureau of Economic Research, New York.

Burns A.F., and Mitchell, W.C. (1946). Measuring Business Cycles. National Bureau of Economic Research, New York.

de Jong, P., and Shephard, N.G. (1995), "The simulation smoother for time series models", Biometrika, 82, 339-350.

Doornik, J.A. (2001), Ox. An Object-Oriented Matrix Programming Language, Timberlake Consultants Press, London.

Durbin, J., and Koopman, S.J. (2001), Time Series Analysis by State Space Methods, Oxford University Press, Oxford, UK.

Fagan, G., Henry, J. and Mestre, R. (2001), An area-wide model (AWM) for the Euro area", ECB Working Paper, No. 42, 
Harding, D. and Pagan, A. (2001), "Extracting, Analysing and Using Cyclical Information, mimeo, August.

Harding, D. and Pagan, A. (2002), "Synchronization of Cycles, University of Melbourne, mimeo, version 6, presented at the conference "Common Features in Rio", Rio de Janeiro, 29th-31st July 2002.

Harvey, A.C. (1989), Forecasting, Structural Time Series and the Kalman Filter, Cambridge University Press, Cambridge, UK.

Harvey, A.C. and Koopman. S.J., (1992), "Diagnostic Checking of Unobserved Components Time Series Models", Journal of Business and Economic Statistics, 10, 377-389.

Harvey, A.C., and Trimbur, T. (2002), "General model-based filters for extracting trends and cycles in economic time series", Review of Economics and Statistics (to appear).

Hodrick R.J., and Prescott, E.C. (1997), "Postwar U.S. Business Cycles: an Empirical Investigation”, Journal of Money, Credit and Banking, 29, 1-16.

Kaiser, R. and Maravall, A. (2001), Measuring Business Cycles in Economic Time Series, Lecture Notes in Statistics, Springer.

Kitagawa, G., and Gersch, W. (1996) Smoothness Priors Analysis of Time Series. New York, Springer-Verlag.

Koopman S.J., Shepard, N., and Doornik, J.A. (1999), "Statistical algorithms for models in state space using SsfPack 2.2”, Econometrics Journal, 2, 113-166.

McDermott, C.J. and Scott, A. (1999) Concordance in business cycles, Reserve Bank of New Zealand Discussion Papers, G99/7

Pagan, A. (2002), Lectures on the business cycle, mimeo, EUI, Florence, April.

Proietti T. (1999), Characterising Business Cycle Asymmetries by Smooth Transition Structural Time Series Models, Studies in Nonlinear Dynamics and Econometrics, Volume 3.3, 141-156, MIT Press, Cambridge, MA. 
Proietti T., Musso A. and Westermann T. (2002). "Estimating Potential Output and the Output gap for the Euro Area: a Model-Based Production Function Approach. EUI Working paper $\mathrm{ECO} / 9 / 2002$.

Stock J.H., and Watson, M. (2002), "Has the business cycle Changed and Why?, mimeo, March.

Whittle, P. (1963), Prediction and Regulation by Linear Least-Squares Methods, London, English Universities Press. 


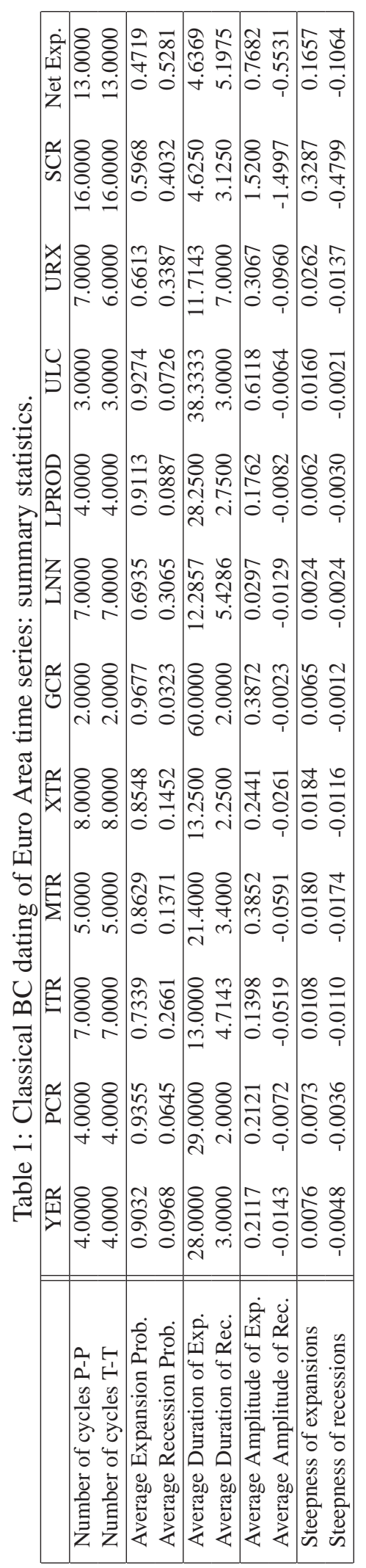




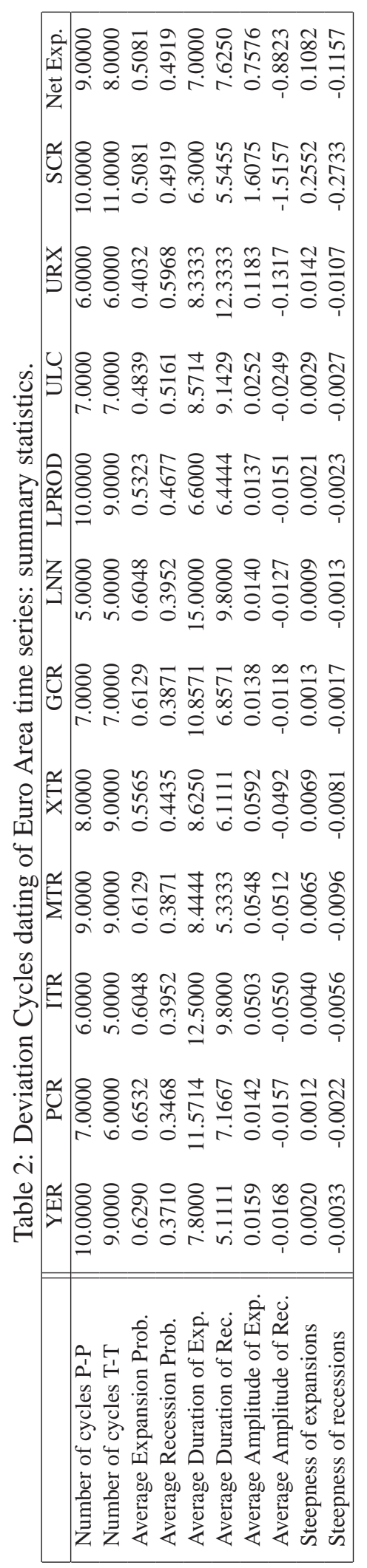


Table 3: Classical BC: Standardised Concordance Index.

\begin{tabular}{|l||cccccc|}
\hline \multicolumn{1}{|c||}{} & EA & D & UK & F & I & US \\
\hline EA & - & 7.15 & 2.48 & 6.29 & 6.35 & 3.40 \\
D & 7.15 & - & 1.93 & 5.41 & 5.43 & 4.43 \\
UK & 2.48 & 1.93 & - & 3.00 & 2.33 & 3.50 \\
F & 6.29 & 5.41 & 3.00 & - & 4.59 & 1.92 \\
I & 6.35 & 5.43 & 2.33 & 4.59 & - & 3.20 \\
US & 3.40 & 4.43 & 3.50 & 1.92 & 3.20 & - \\
\hline
\end{tabular}

Table 4: Test for BC independence using HAC standard errors (Newey-West estimator with truncation parameter equal to 5).

\begin{tabular}{|l||rrrrrr|}
\hline & EA & D & UK & F & I & US \\
\hline EA & - & 52.52 & 1.80 & 4.41 & 12.15 & 2.62 \\
D & 7.85 & - & 1.53 & 3.07 & 6.73 & 3.33 \\
UK & 1.87 & 1.66 & - & 1.90 & 2.37 & 4.25 \\
F & 10.47 & 9.02 & 1.89 & - & 5.49 & 1.51 \\
I & 4.86 & 4.79 & 1.82 & 2.94 & - & 2.57 \\
US & 3.02 & 4.02 & 2.65 & 1.52 & 3.70 & - \\
\hline
\end{tabular}

Table 5: Deviation cycles: Standardised Concordance Index.

\begin{tabular}{|l||cccccc|}
\hline & EA & D & UK & F & I & US \\
\hline EA & - & 4.83 & 3.42 & 4.71 & 5.77 & 2.75 \\
D & 4.83 & - & 2.95 & 2.66 & 3.48 & 2.53 \\
UK & 3.42 & 2.95 & - & 2.07 & 2.33 & 2.26 \\
F & 4.71 & 2.66 & 2.07 & - & 3.67 & 2.47 \\
I & 5.77 & 3.48 & 2.33 & 3.67 & - & 1.90 \\
US & 2.75 & 2.53 & 2.26 & 2.47 & 1.90 & - \\
\hline
\end{tabular}

Table 6: Test for deviation cycle independence using HAC standard errors (Newey-West estimator with truncation parameter equal to 5).

\begin{tabular}{|l||rrrrrr|}
\hline & EA & D & UK & F & I & US \\
\hline EA & - & 15.27 & 4.96 & 12.93 & 11.12 & 4.45 \\
D & 8.89 & - & 2.06 & 4.53 & 4.49 & 2.75 \\
UK & 3.68 & 2.14 & - & 5.39 & 3.33 & 6.56 \\
F & 8.38 & 4.68 & 4.91 & - & 4.81 & 2.87 \\
I & 13.02 & 6.30 & 5.55 & 5.32 & - & 3.22 \\
US & 3.60 & 3.78 & 4.27 & 2.28 & 2.79 & - \\
\hline
\end{tabular}




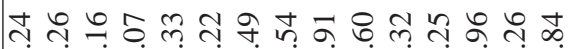

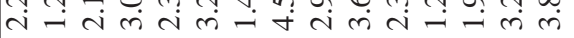

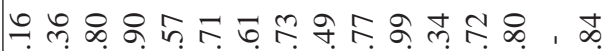
-4तis

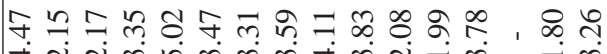

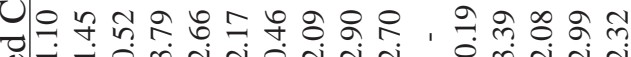

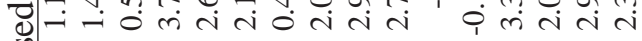

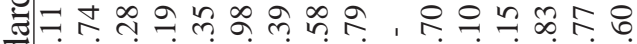

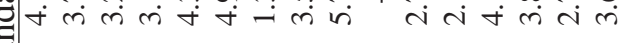

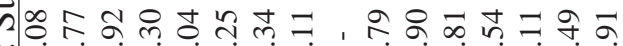

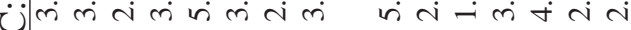
๑

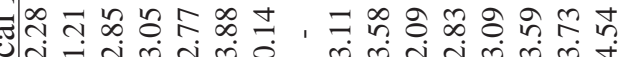
त-तलतल लmततलmल

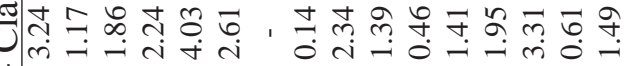
约

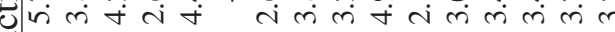

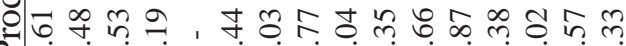
중

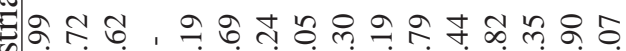

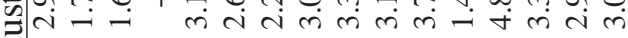
ق

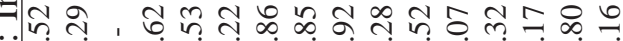

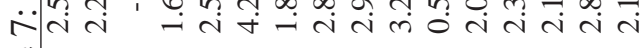
$\because$

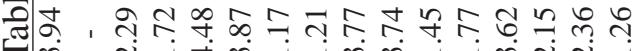

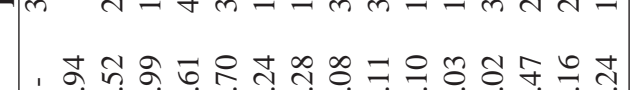

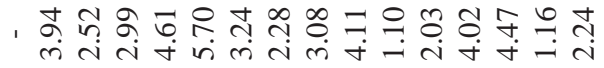

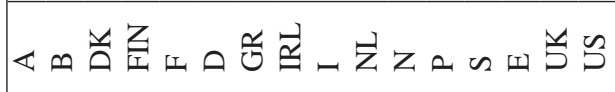




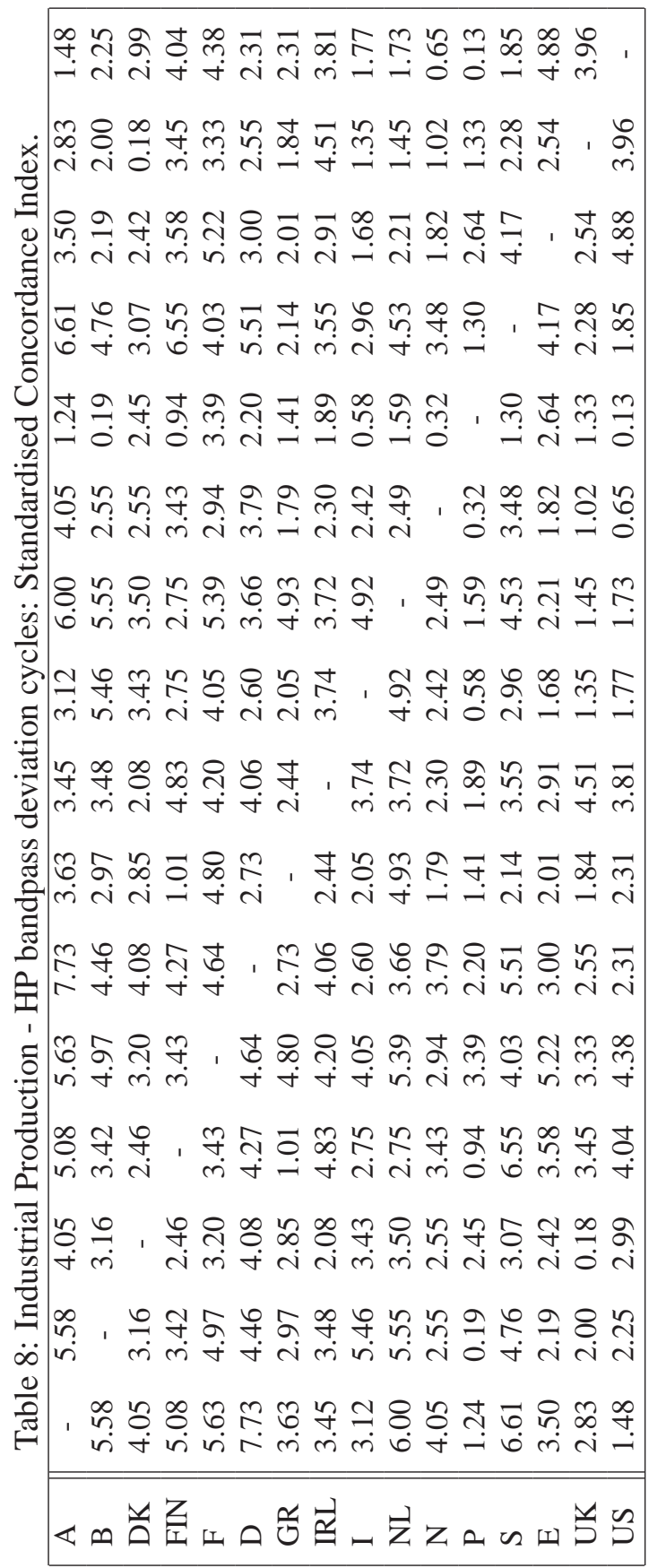



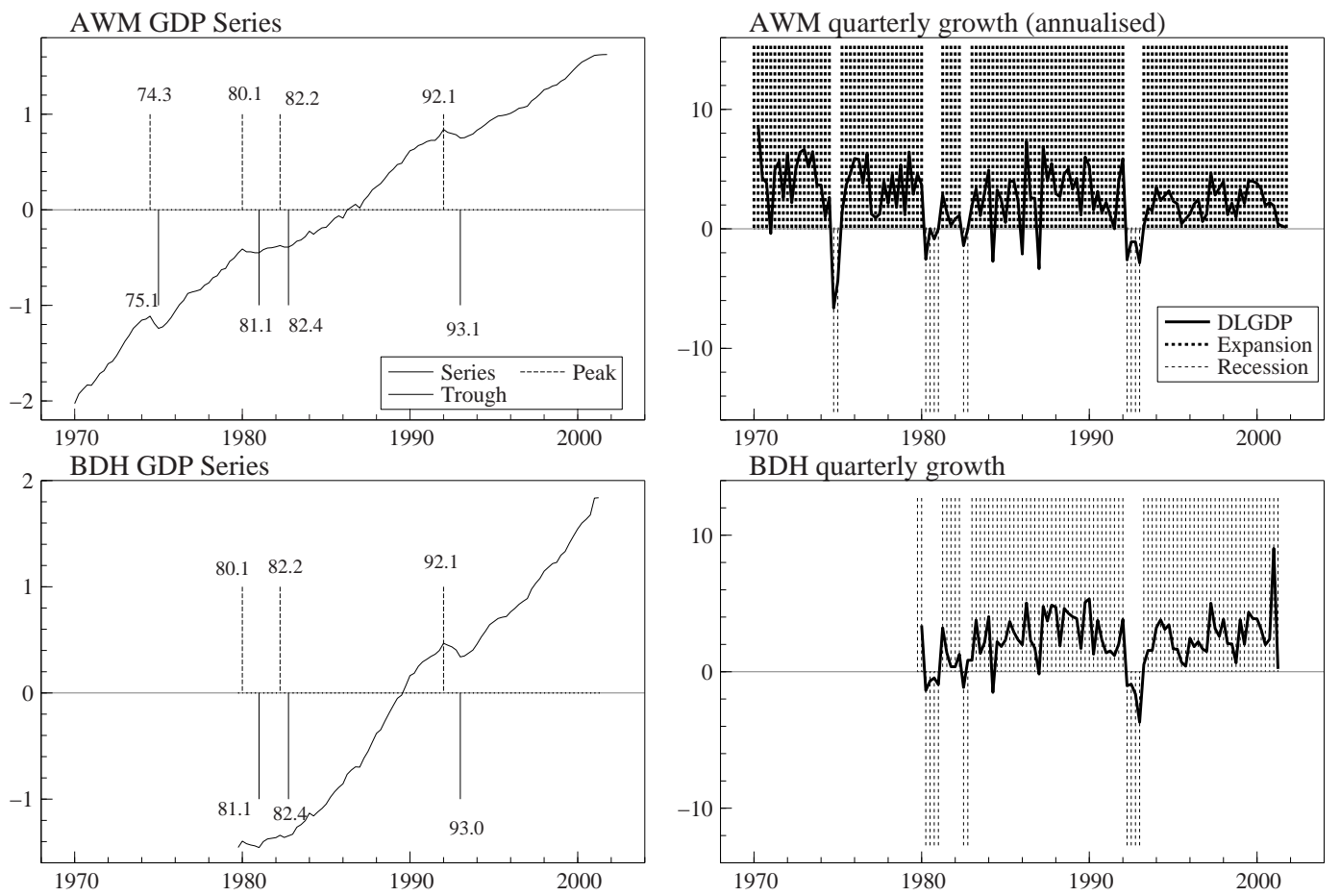

Figure 1: Classical cycle turning points, expansions and recessions, in the Euro area quarterly real GDP (seasonally adjusted, logarithms); ECB series and Beyer, Doornik and Hendry (2000) estimates. 

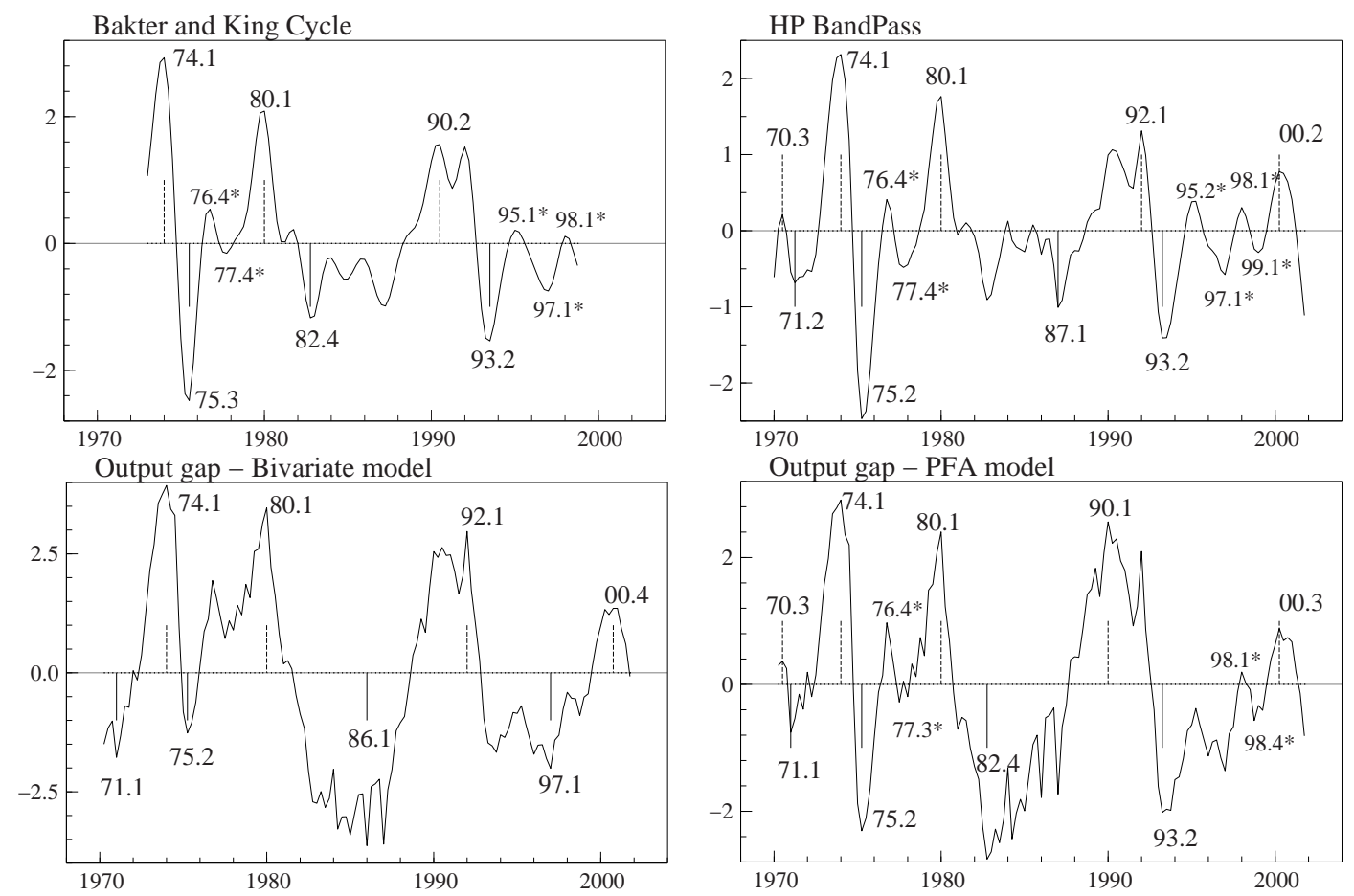

Figure 2: Turning points for four alternative measures of the Euro Area deviation cycle. An asterisk (*) denotes a turning point that was censored according to amplitude considerations (see text for details). 

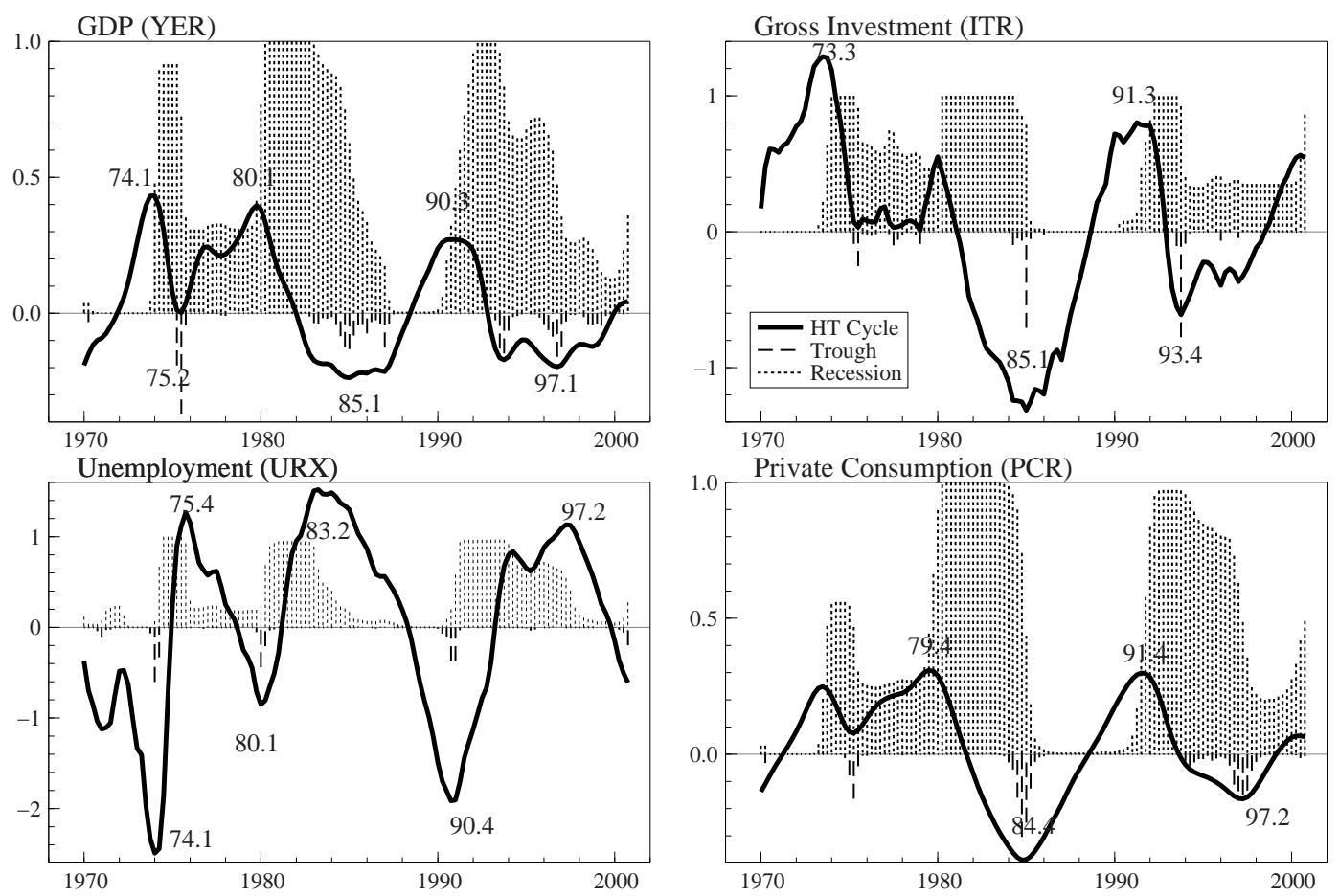

Figure 3: Harvey and Trimbur (2002) deviation cycles, recession probabilities, and trough probabilities (inverted scale), for selected Euro Area series. 

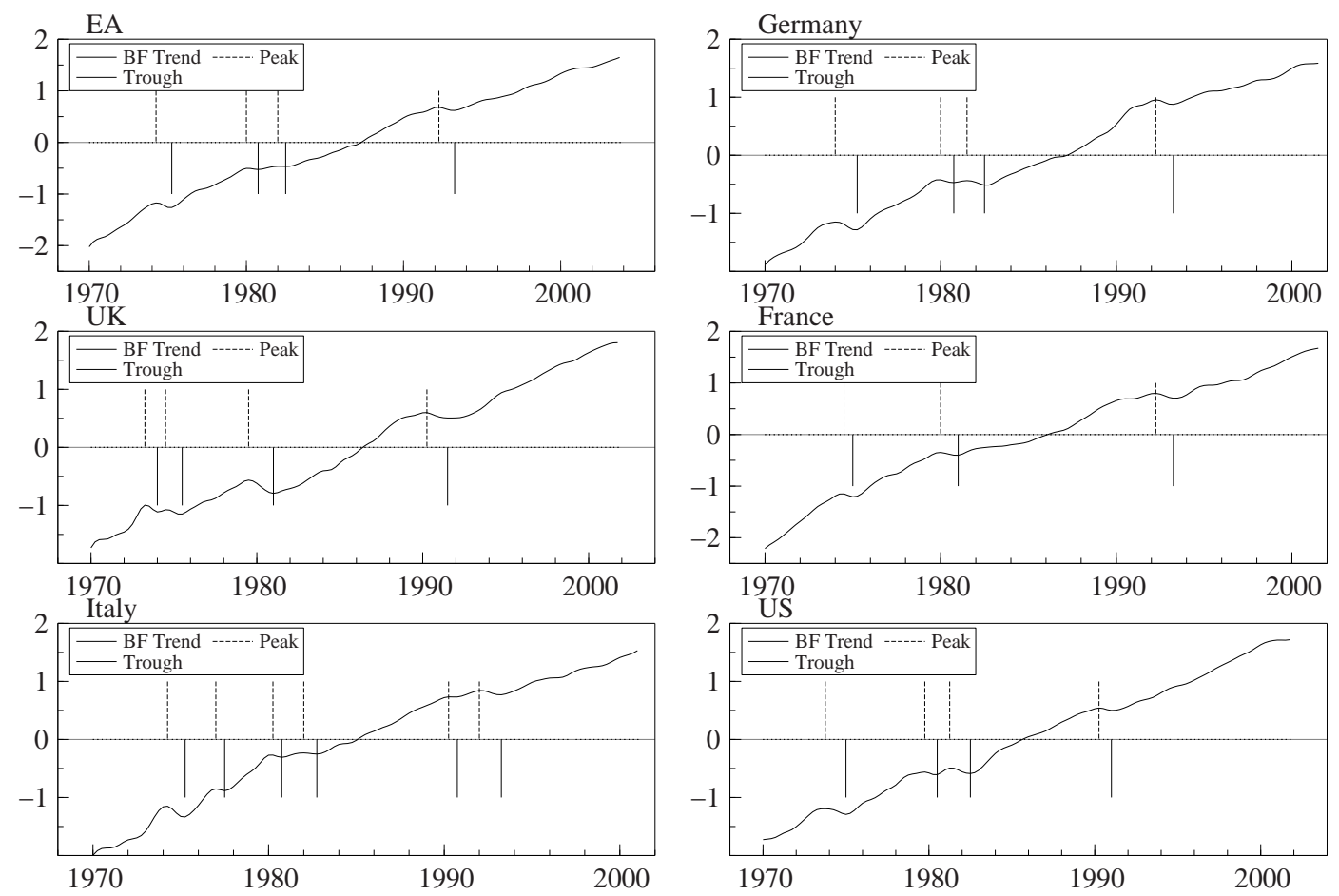

Figure 4: Classical cycle turning points for EA, Germany, France, Italy, UK and the USA, based on $\mathrm{HP}(1.25)$ filtered quarterly real GDP. 

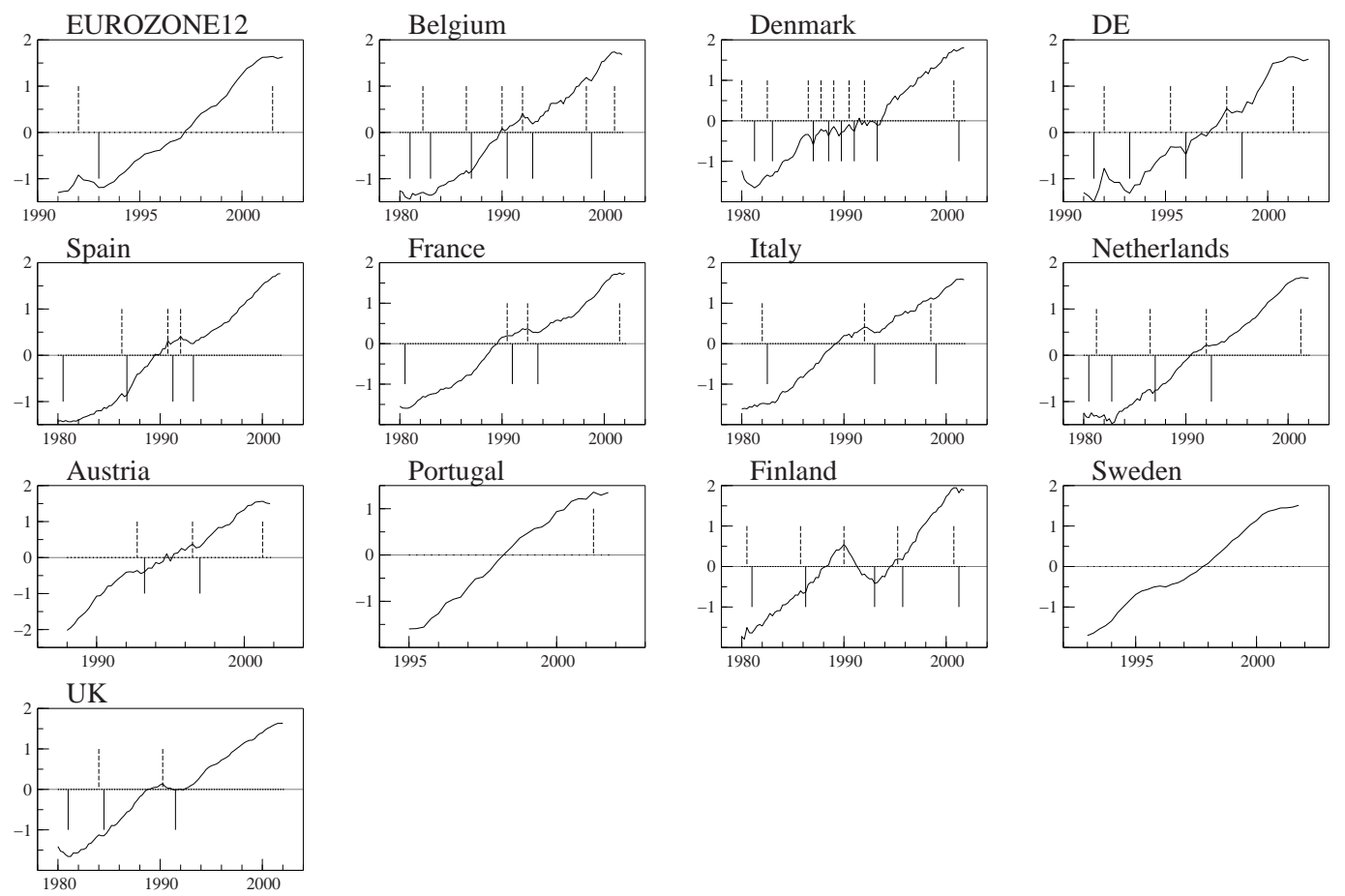

Figure 5: Classical cycle turning points, for the Euro zone countries based on quarterly real GDP (seasonally adjusted, logarithms); Eurostat series, 1980.1-2002.1. 

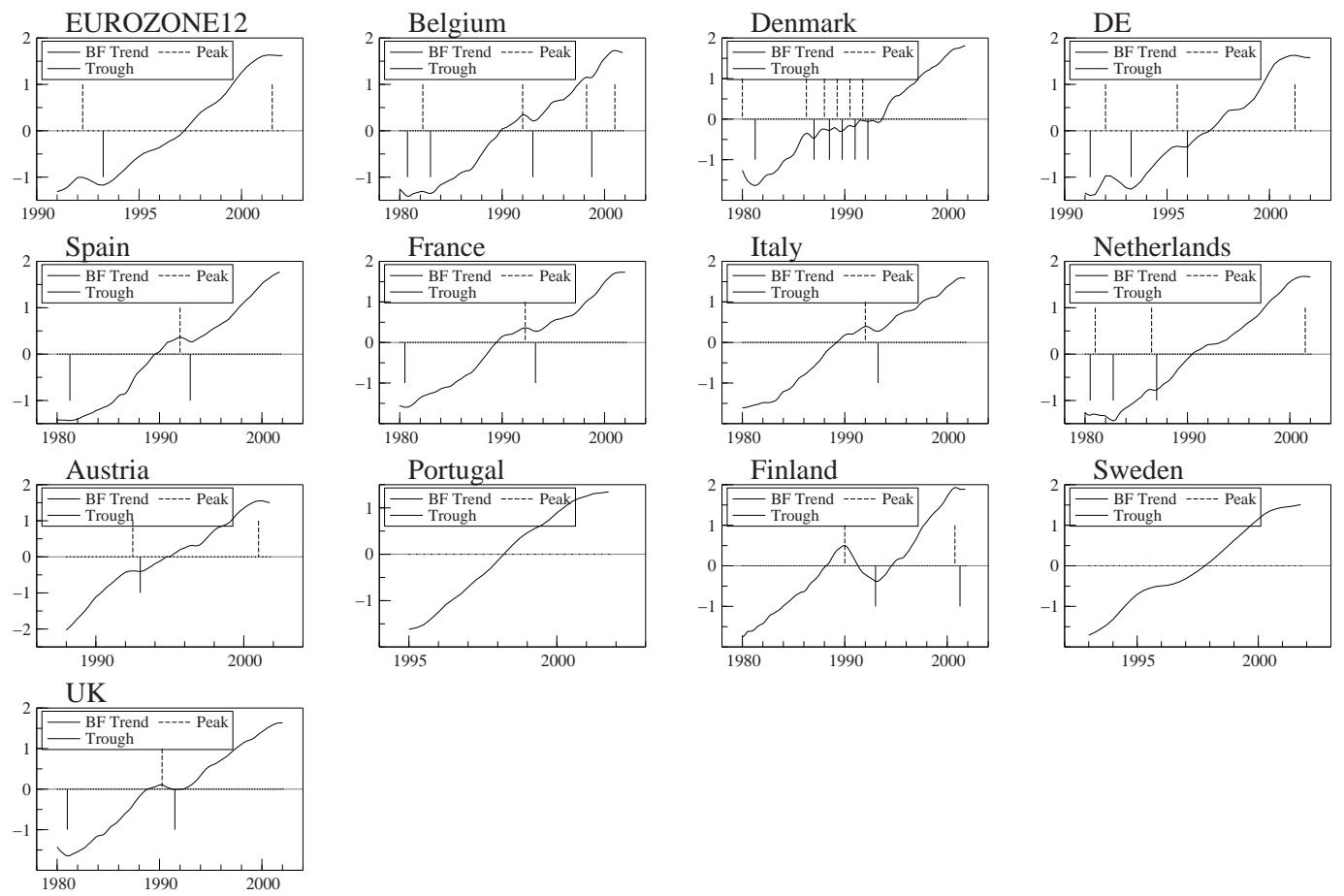

Figure 6: Classical cycle turning points, for the Euro zone countries based on estimates of a trend resulting from $\mathrm{HP}(1.25)$. 

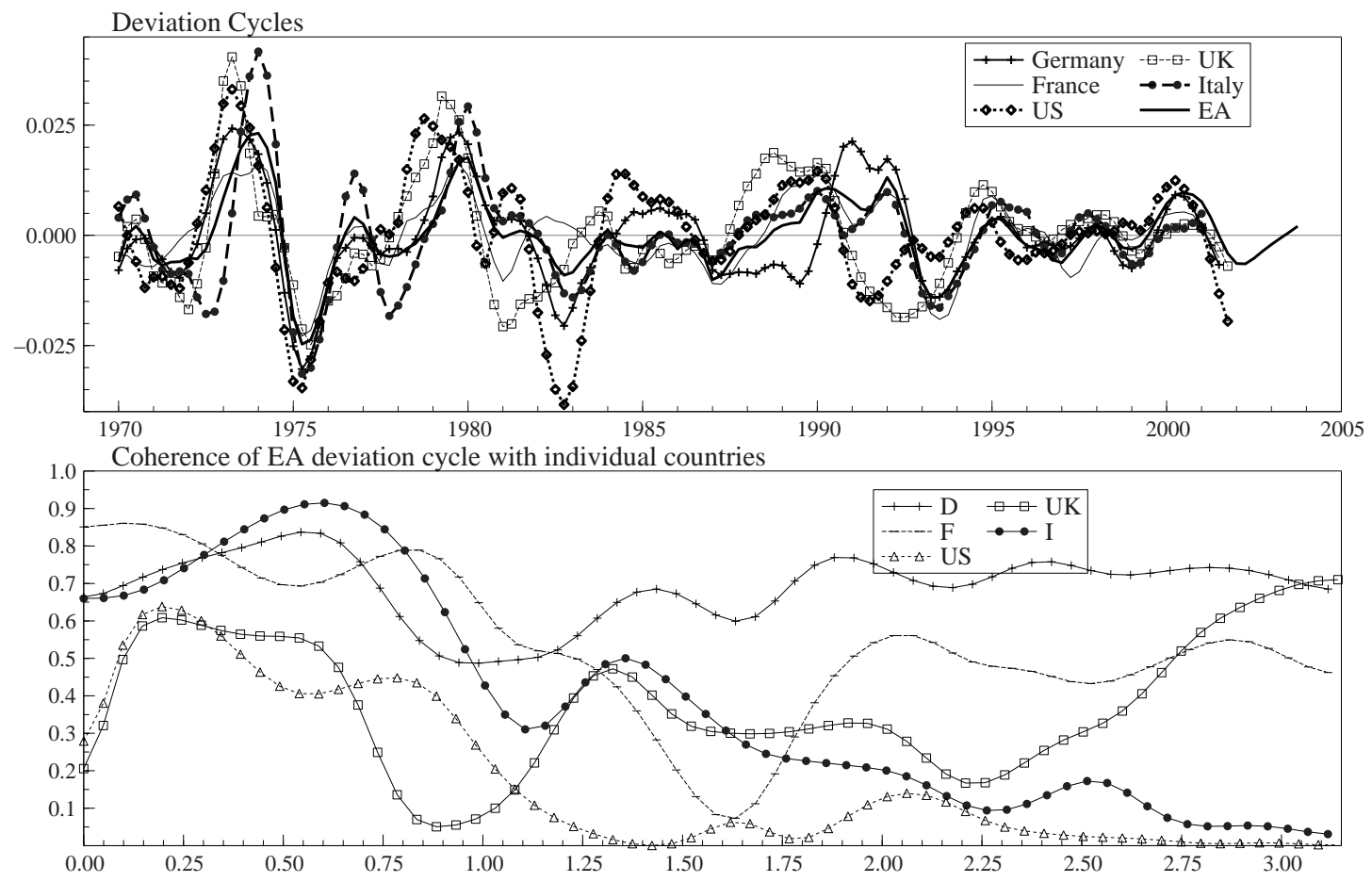

Figure 7: Coherence of the Euro Area HPB deviation cycle and those of selected countries. 

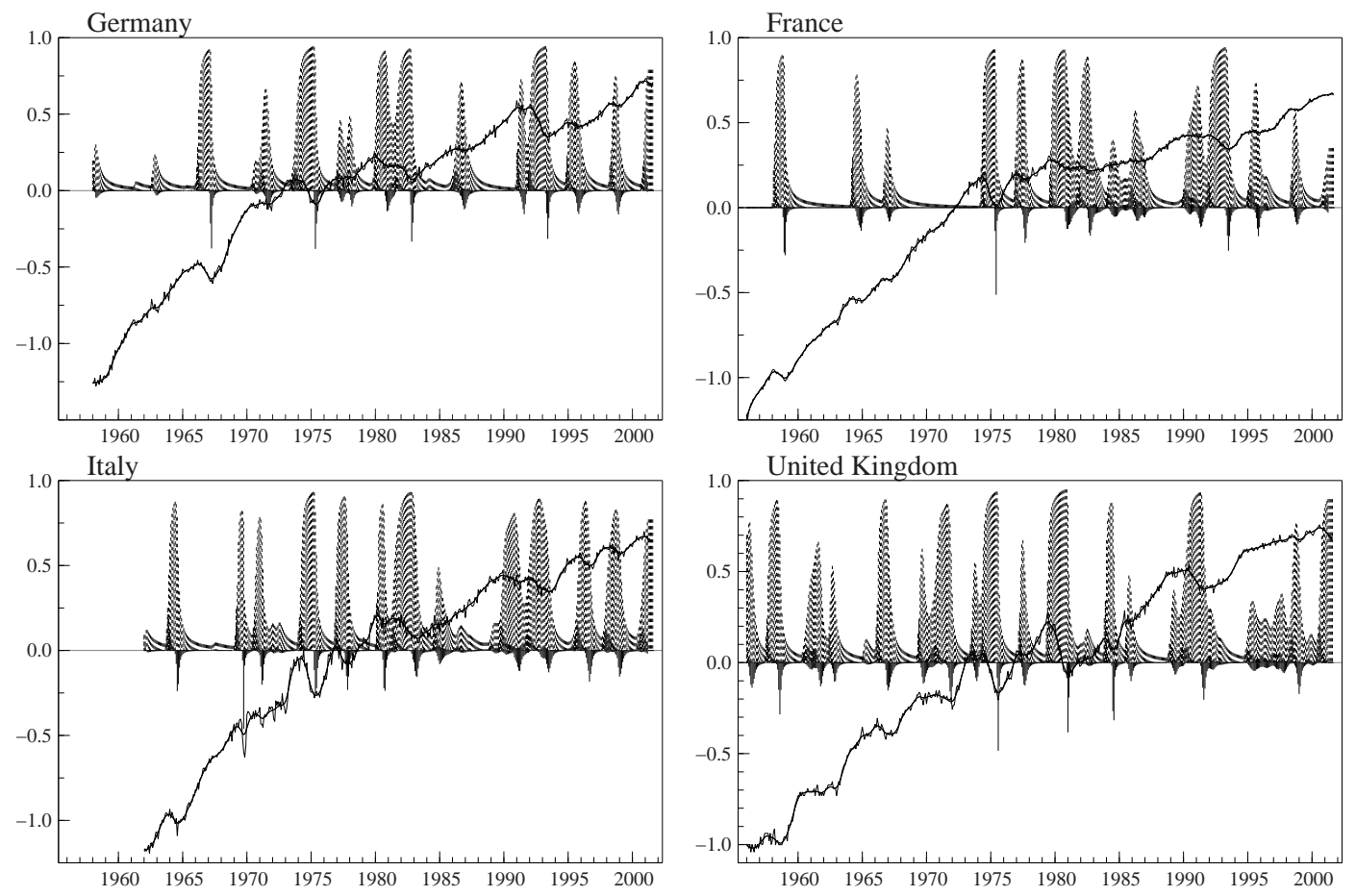

Figure 8: Classical BC dating of monthly industrial production based on $\operatorname{HP}(1.25)$; recession and turning points probabilities. 

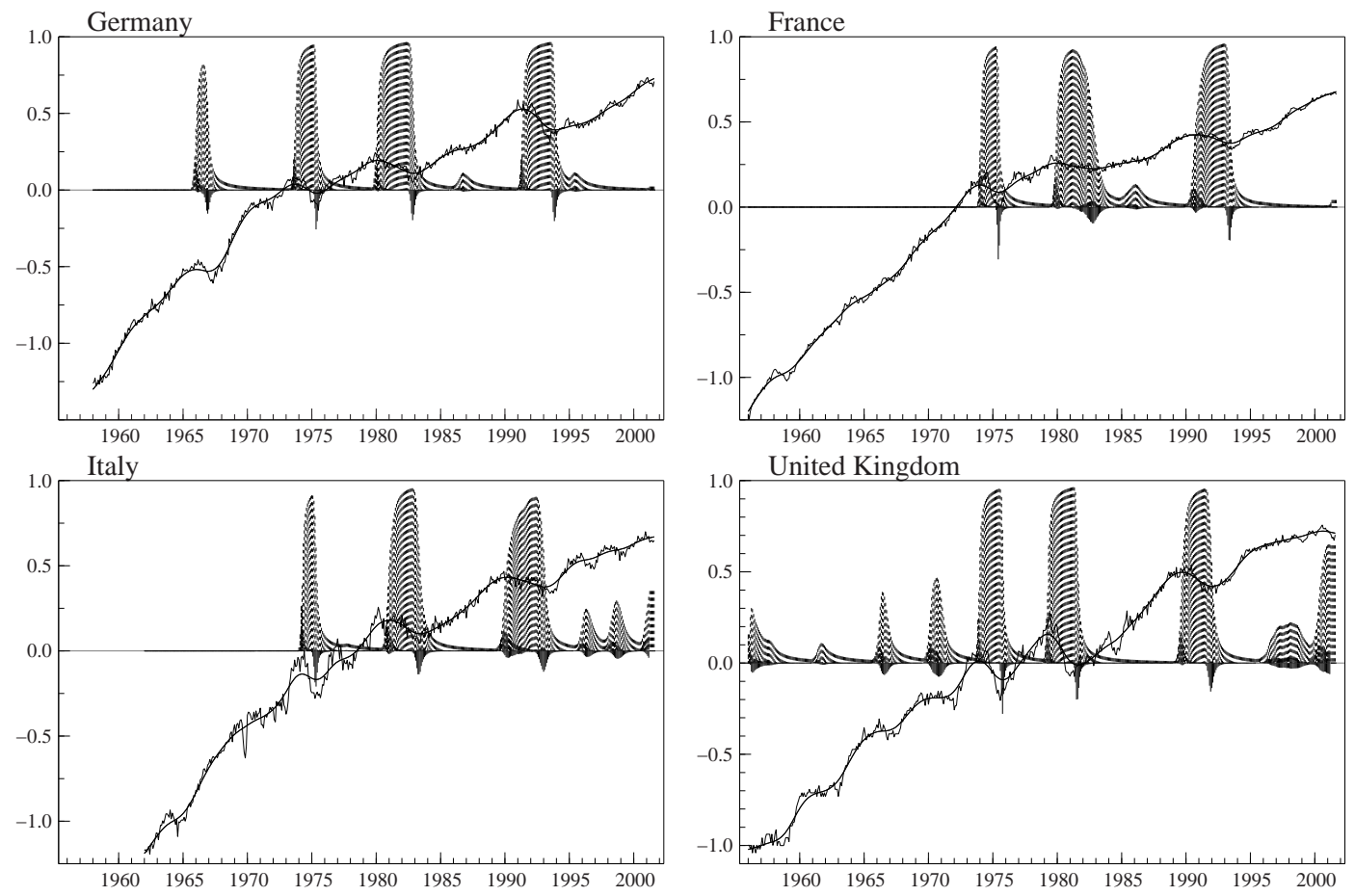

Figure 9: Classical BC dating of monthly industrial production based on $\mathrm{HP}(4)$; recession and turning points probabilities. 


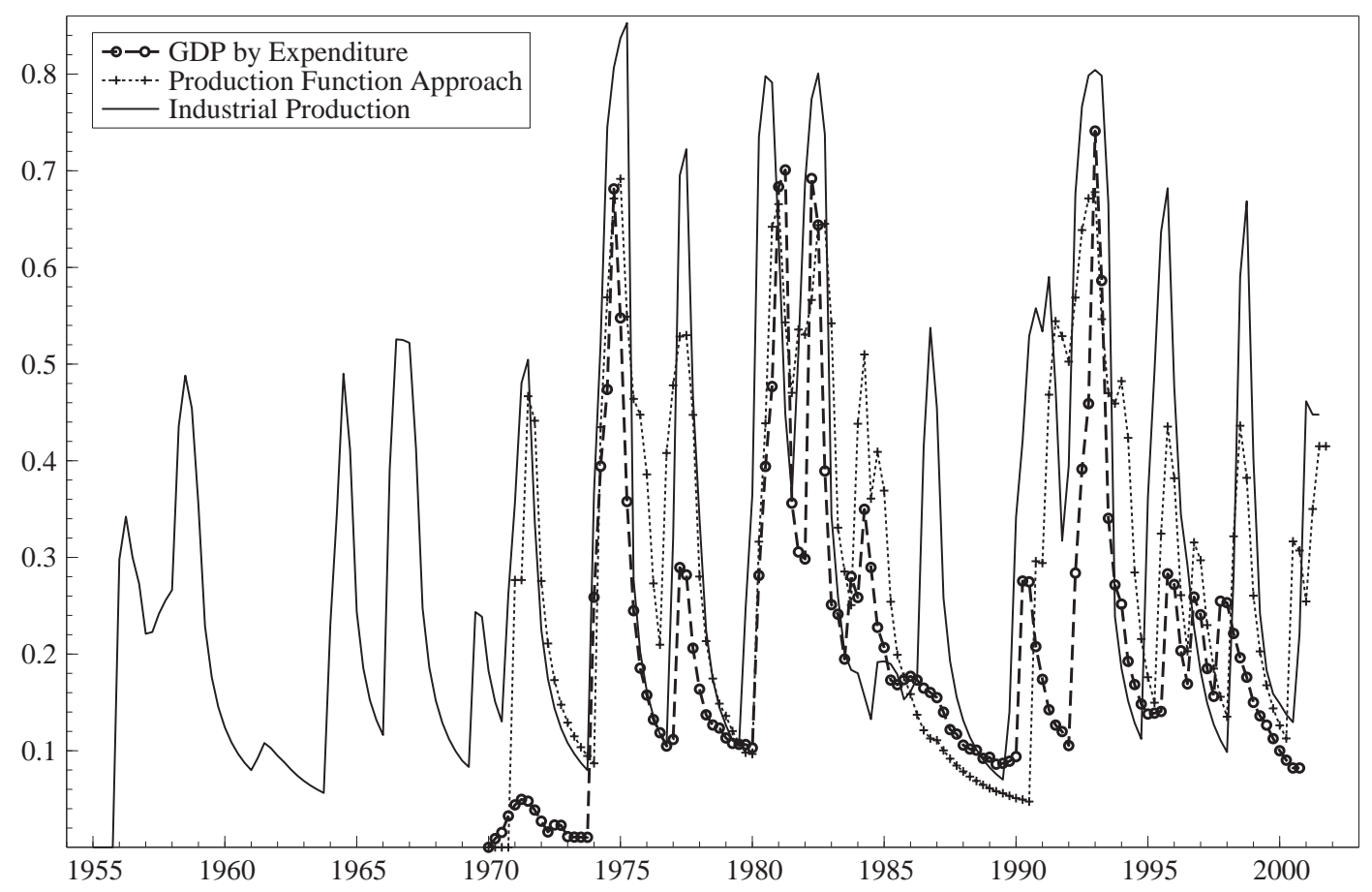

Figure 10: Three sets of diffusion indices for classical business cycles in the Euro area. 

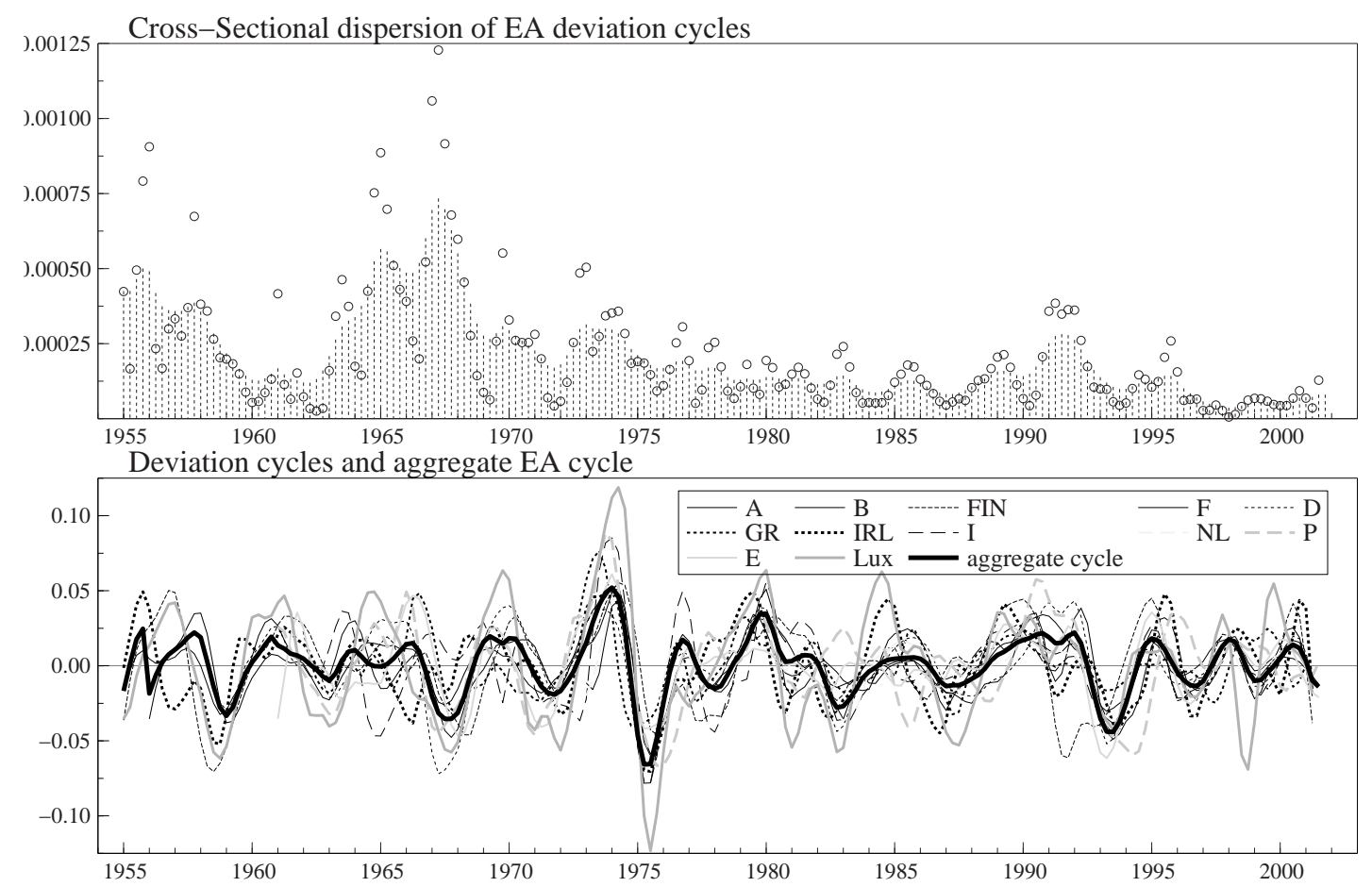

Figure 11: Cross-sectional dispersion of HP bandpass deviation cycles for EA member countries industrial production series. 


\section{A The Algorithm for Dating the Business Cycle}

For exposition sake we illustrate the dating algorithm with reference to the quarterly case. A few details concerning the monthly case are deferred to appendix A.5. Despite the apparent complexity of the algorithm, its implementation requires less than 70 lines of $\mathrm{Ox}$ code ${ }^{3}$.

\section{A.1 The underlying Markov Chain}

At any time $t$ the economy can be in either of two mutually esclusive states or phases: expansion $\left(E_{t}\right)$ or recession $\left(R_{t}\right)$. A peak terminates an expansion, whereas a trough terminates a recession. For the imposition of minimum duration constraint and to enforce the alternation of peaks and troughs, it is useful to distinguish turning points within the two basic states, by posing:

$$
\mathrm{E}_{t} \equiv \begin{cases}\mathrm{EC}_{t} & \text { Expansion Continuation } \\ \mathrm{R}_{t} & \text { Peak } \\ \mathrm{RC}_{t} & \text { Recession Continuation } \\ \mathrm{T}_{t} & \text { Trough }\end{cases}
$$

From $\mathrm{EC}_{t}$ we can make a transition to $\mathrm{P}_{t+1}$ or continuate the expansion $\left(\mathrm{EC}_{t} \rightarrow \mathrm{EC}_{t+1}\right)$, but not viceversa, since only $\mathrm{P}_{t} \rightarrow \mathrm{RC}_{t+1}$ is admissible. Analogously, from $\mathrm{RC}_{t}$ we can visit either $\mathrm{RC}_{t+1}$ or $\mathrm{T}_{t+1}$, but from $\mathrm{T}_{t}$ we move to $\mathrm{EC}_{t+1}$ with probability 1 .

Denoting by $p_{E P}=P\left(\mathrm{P}_{t+1} \mid \mathrm{EC}_{t}\right)$ the probability of making a transition to a peak within an expansionary pattern, $p_{E E}=P\left(\mathrm{EC}_{t+1} \mid \mathrm{EC}_{t}\right)=1-p_{E P}$, and analogously $p_{R T}=P\left(\mathrm{~T}_{t+1} \mid \mathrm{RC}_{t}\right)$, $p_{R R}=P\left(\mathrm{RC}_{t+1} \mid \mathrm{RC}_{t}\right)=1-p_{R T}$, we define a first order Markov chain (MC) with four states, denoted $S_{t}$, with transition matrix:

\begin{tabular}{|l|cccc|}
\hline & $\mathrm{EC}_{t+1}$ & $\mathrm{P}_{t+1}$ & $\mathrm{RC}_{t+1}$ & $\mathrm{~T}_{t+1}$ \\
\hline $\mathrm{EC}_{t}$ & $p_{E E}$ & $p_{E P}$ & 0 & 0 \\
$\mathrm{P}_{t}$ & 0 & 0 & 1 & 0 \\
$\mathrm{RC}_{t}$ & 0 & 0 & $p_{R R}$ & $p_{R T}$ \\
$\mathrm{~T}_{t}$ & 1 & 0 & 0 & 0 \\
\hline
\end{tabular}

\footnotetext{
${ }^{3}$ All the computations in the paper were performed using the object oriented matrix programming language Ox 3.0 by Doornik (2001), and the library of state space function SsfPack 2.3 by Koopman et al. (1999).
} 
The dating rules impose ties on the minimum duration of a phase, which amounts to two quarters, and this is automatically enforced in the quarterly case by our four states characterisation (as a matter of fact $\left\{\mathrm{EC}_{t-1}, \mathrm{P}_{t}\right\}$, complies with this requirement, since both events belong to the expansionary phase; similarly a trough cannot occur immediately after a peak), and on the minimum duration of a full cycle. The latter is defined in terms of peak-to-peak or trough-to-trough patterns and amounts to five quarters, as a direct transposition of the Bry and Boschan rule to the quarterly case. Thus the pattern $\left\{\mathrm{T}_{t-4}, \mathrm{EC}_{t-3}, \mathrm{P}_{t-2}, \mathrm{RC}_{t-1}, \mathrm{~T}_{t}\right\}$ is not admissible. The minimum duration constraint are important for the characterisation of the chain: that imposed on the full cycle duration determines the order of the $\mathrm{MC}$, whereas that imposed on the phases length determines the number of admissible states.

The tie on the full cycle yields a 5th order MC that can be converted to a first order one by combining elements of the original chain, $S_{t}$. The states of the derived MC are defined by appropriately combining the original ones into

$$
S_{t}^{*}=\left\{S_{t-4}, S_{t-3}, S_{t-2}, S_{t-1}, S_{t}\right\}
$$

The ties however reduce the number of states to 24 . These are listed in table 9: the first column labels the states and the second spells out how they are formed by combining the elementary states of the original MC. The last two columns indicate the states to which a transition is admissible (two at most) and the associated transition probability. The transition matrix is thus immediately derived from the above table. It should be noticed that all the states ending with a peak or a trough must visit certain states with probability one.

The two parameters $p_{E P} p_{R T}$ uniquely specify the Markov chain. In the next section we show how these are computed with the support of a time series or a stochastic process. As a matter of fact, the dating algorithm is completed by establishing rules for scoring the transition probabilities. We conclude this section discussing how the two elementary transition probabilities influence the features of the chain, by deriving the ergodic probabilities of expansions and recessions and those of peaks and troughs: these are easily computed from the ergodic probabilities of the 5 th order $\mathrm{MC}$, by marginalising previous states.

The following table provides the ergodic expansion probabilites for different values of $p_{R T}$ and $p_{E P}:$ 


\begin{tabular}{|l|llll|}
\hline & \multicolumn{4}{|c|}{$p_{R T}$} \\
$p_{E P}$ & 0.05 & 0.15 & 0.25 & 0.35 \\
\hline 0.05 & 0.50 & 0.73 & 0.81 & 0.85 \\
0.15 & 0.27 & 0.50 & 0.61 & 0.67 \\
0.25 & 0.19 & 0.39 & 0.50 & 0.57 \\
0.35 & 0.15 & 0.33 & 0.43 & 0.50 \\
\hline
\end{tabular}

As far as the ergodic recession probabilities are concerned, these are obtained by transposing the table. When $p_{R T}=p_{E P}$, these probabilities always equal $1 / 2$.

The ergodic probabilities of a peak are presented in the table below. It should be noticed that the table is symmetric, a fact that underlies a major implication of the chain, namely that the probability of a peak is equal to that of a trough.

\begin{tabular}{|l|llll|}
\hline & \multicolumn{4}{|c|}{$p_{R T}$} \\
$p_{E P}$ & 0.05 & 0.15 & 0.25 & 0.35 \\
\hline 0.05 & 0.02 & 0.03 & 0.04 & 0.04 \\
0.15 & 0.03 & 0.06 & 0.08 & 0.08 \\
0.25 & 0.04 & 0.08 & 0.10 & 0.11 \\
0.35 & 0.04 & 0.08 & 0.11 & 0.12 \\
\hline
\end{tabular}

Also, these probabilities do not change sensibly as either $p_{R T}$ or $p_{E P}$ or both are increased: this stems from the minimum duration constraints that limit the number of turning points.

\section{A.2 Scoring the transition probabilities}

As seen in the previous section, the characterisation of the phases of the business cycle and the duration constraints define an underlying MC that is fully specified once the core parameters, $p_{R T}$ and $p_{E P}$ are known. These can be estimated by maximum likelihood techniques from an observed time series in a model based framework, if it is assumed that the latter is a realisation of a stochastic process that is dependent upon the state of the economy as represented by the chain. This idea is at the foundation of the class of Markov-Switching models, that postulate that the growth rate and/or the innovation variance and/or the transmission mechanism vary according to recessions and expansions. 
In this paper we consider the alternative strategy of scoring the two parameters according to patterns in the series, $y_{t}$. There are several ways of doing so, with different degrees of complexity, but we will concentrate on the BBQ rule by Harding and Pagan (2001). Other, simpler, popular rules are the calculus and the Okun's rule.

According to the BBQ dating rule, we define an expansion termination sequence, $\mathrm{ETS}_{t}$, and a recession terminating sequence, $\mathrm{RTS}_{t}$, respectively as:

$$
\begin{aligned}
& \text { ETS }_{t}=\left\{\left(\Delta y_{t+1}<0\right) \cap\left(\Delta_{2} y_{t+2}<0\right)\right\} \\
& \text { RTS }_{t}=\left\{\left(\Delta y_{t+1}>0\right) \cap\left(\Delta_{2} y_{t+2}>0\right)\right\}
\end{aligned}
$$

The former defines a candidate point for a peak, which terminates the expansion, whereas the latter defines a candidate for a trough.

The joint distribution of the sequences $\left\{\mathrm{ETS}_{t}, \mathrm{RTS}_{t}, t=1, \ldots, T\right\}$ depends on the stochastic process generating the available series and is usually analitically intractable due to the presence of serial correlation and the mutually non exclusive nature of the termination sequences. As regards the latter, denoting by $\overline{\mathrm{ETS}}_{t}$ the complementary event of ETS,$\overline{\mathrm{RTS}}_{t}$ that of $\mathrm{RTS}_{t}$ and defining $\mathcal{P}_{t}^{(E T S)}=P\left(\mathrm{ETS}_{t}\right), \mathcal{P}_{t}^{(R T S)}=P\left(\mathrm{RTS}_{t}\right)$, at time $t$ the joint probability distribution of the possible events is provided by the following table:

\begin{tabular}{l|cc|c} 
& ETS $_{t}$ & $\overline{\text { ETS }_{t}}$ & Marginal \\
\hline RTS $_{t}$ & 0 & $\mathcal{P}_{t}^{(R T S)}$ & $\mathcal{P}_{t}^{(R T S)}$ \\
$\overline{\text { ETS }}_{t}$ & $\mathcal{P}_{t}^{(E T S)}$ & $1-\mathcal{P}_{t}^{(E T S)}-\mathcal{P}_{t}^{(R T S)}$ & $1-\mathcal{P}_{t}^{(R T S)}$ \\
\hline Marginal & $\mathcal{P}_{t}^{(E T S)}$ & $1-\mathcal{P}_{t}^{(E T S)}$ & 1
\end{tabular}

whence it can be seen that $\mathrm{ETS}_{t}$ and RTS cannot both be true at the same time.

Serial correlation complicates the computation of $\mathcal{P}_{t}^{(E T S)}$ and $\mathcal{P}_{t}^{(R T S)}$, since the terminating sequences are not independent of their past; furthermore it must be stressed that the BBQ rule induces autocorrelation itself, that is even if $\Delta y_{t} \sim \operatorname{NID}\left(\mu, \sigma^{2}\right)$, e.g. $y_{t}$ is a random walk, $\left\{\mathrm{ETS}_{t}, \mathrm{RTS}_{t}, t=1, \ldots, T\right\}$ will be autocorrelated. Therefore it seems that the only way to go about the characterisation of business cycle for a particular stochastic process is stochastic simulation.

Let us return to the non parametric scoring of the transition probabilities according to the available time series. If at time $t$ the chain $S_{t}^{*}$ is in any of the expansionary states for which a 
transition to a peak is possible and an expansion terminating sequence occurs at time $t+1$, i.e ETS $_{t+1}$ is true, then we move to a new state $S_{t+1}^{*}$, such that $S_{t+1}=\mathrm{P}_{t+1}$ and the previous four elementary states are common to the last four in $S_{t}^{*}$.

It is useful at this point to classify the states of $S_{t}^{*}$ by defining the sets:

$\mathcal{S}_{E P}=\left\{S_{3}^{*}, S_{4}^{*}, S_{5}^{*}, S_{9}^{*}, S_{19}^{*}, S_{20}^{*}, S_{22}^{*}\right\}$ defines the set of states featuring an expansionary state at time $t\left(S_{t}=\mathrm{EC}_{t}\right)$ and that are available for a transition to a peak.

$\mathcal{S}_{P}=\left\{S_{6}^{*}, S_{10}^{*}, S_{21}^{*}, S_{23}^{*}\right\}$ defines the set of states featuring a peak at time $t\left(S_{t}=\mathrm{P}_{t}\right)$.

$\mathcal{S}_{R T}=\left\{S_{1}^{*}, S_{7}^{*}, S_{8}^{*}, S_{11}^{*}, S_{12}^{*}, S_{14}^{*}, S_{17}^{*}\right\}$ defines the set of states featuring a recessionary state at time $t\left(S_{t}=\mathrm{RC}_{t}\right)$ and that are available for a transition to a trough.

$\mathcal{S}_{T}=\left\{S_{2}^{*}, S_{13}^{*}, S_{15}^{*}, S_{18}^{*}\right\}$ defines the set of states featuring a peak at time $t\left(S_{t}=\mathrm{P}_{t}\right)$.

The set of expansionary states, $\mathcal{S}_{E}$, is the union of $\mathcal{S}_{E P}, \mathcal{S}_{P}$ and $S_{16}^{*}$, in symbols:

$$
\mathcal{S}_{E}=\mathcal{S}_{E P} \cup \mathcal{S}_{P} \cup S_{16}^{*}
$$

The set of recessionary states, $\mathcal{S}_{R}$, is the union of $\mathcal{S}_{R T}, \mathcal{S}_{T}$ and $S_{24}^{*}$, in symbols:

$$
\mathcal{S}_{R}=\mathcal{S}_{R T} \cup \mathcal{S}_{T} \cup S_{24}^{*}
$$

The scoring rules are then formalised in the following algorithm: 
If $\left\{S_{t}^{*}=s_{E P}, s_{E P} \in \mathcal{S}_{E P}\right\}$ and $\mathrm{ETS}_{t+1}$ is true, then $\left\{S_{t+1}^{*}=s_{P}, s_{P} \in \mathcal{S}_{P}\right\}$. Hence, the transition probability $p_{E P}$ is computed as:

$$
\begin{aligned}
p_{E P} & =P\left(\left\{S_{t}^{*}=s_{E P}, s_{E P} \in \mathcal{S}_{E P}\right\} \cap \mathrm{ETS}_{t+1}\right) \\
& =\mathrm{I}\left(\mathrm{ETS}_{t+1}\right) \sum_{s_{E P} \in \mathcal{S}_{E P}} P\left(S_{t}^{*}=s_{E P}\right),
\end{aligned}
$$

where $\mathrm{I}(\cdot)$ is the indicator function. Else, if $\mathrm{ETS}_{t+1}$ is false then the expansion is continued, that is $S_{t+1}^{*}=s_{E P}, s_{E P} \in \mathcal{S}_{E P}$; the associated transition probability is $p_{E E}=1-p_{E P}$.

Else, if $\left\{S_{t}^{*}=s_{R T}, s_{R T} \in \mathcal{S}_{R T}\right\}$ and $\mathrm{RTS}_{t+1}$ is true, then $\left\{S_{t+1}^{*}=s_{T}, s_{T} \in \mathcal{S}_{T}\right\}$. Hence, the transition probability $p_{R T}$ is computed as:

$$
\begin{aligned}
p_{R T} & =P\left(\left\{S_{t}^{*}=s_{R T}, s_{R T} \in \mathcal{S}_{R T}\right\} \cap \mathrm{RTS}_{t+1}\right) \\
& =\mathrm{I}\left(\mathrm{RTS}_{t+1}\right) \sum_{s_{R T} \in \mathcal{S}_{R T}} P\left(S_{t}^{*}=s_{R T}\right),
\end{aligned}
$$

Else, if $\mathrm{RTS}_{t+1}$ is false, then the recession is continued, that is $S_{t+1}^{*}=s_{R T}, s_{R T} \in$ $\mathcal{S}_{R T}$; the associated transition probability is $p_{R R}=1-p_{R T}$.

The case when $\mathrm{ETS}_{t+1}$ and $\mathrm{RTS}_{t+1}$ are both false is implicitly covered by the above dating rule. Probabilistic dating based on a maintained stochastic process replaces the indicator function, $\mathrm{I}(\cdot)$, with the probability of the terminating sequences, $\mathcal{P}_{t+1}^{(E T S)}, \mathcal{P}_{t+1}^{(R T S)}$.

Let now $\mathcal{F}_{t}$ denote the collection of $\mathrm{I}\left(\mathrm{ETS}_{j}\right), \mathrm{I}\left(\mathrm{RTS}_{j}\right), j=1,2, \ldots, t$, and let $P\left(S_{t}^{*} \mid \mathcal{F}_{t}\right)$ denote the probability of being in any particular state at time $t$ conditional on this information set. Assuming that this probability is known we can compute recursively the probability of the chain at subsequent times by the following filter:

i. Given the availability of $P\left(S_{t}^{*} \mid \mathcal{F}_{t}\right)$ at time $t$, let us denote by $\pi_{t}^{*}$ the $m \times 1$ vector containing them, with $m=24$ in the quarterly case. Define the two $m \times 1$ selection vectors $v_{E P}$, with ones corresponding to the elements of $\mathcal{S}_{E P}$ and zero otherwise, and $v_{R T}$, with ones corresponding to the elements of $\mathcal{S}_{R T}$ and zero otherwise.

ii. Compute the transition probabilities of the chain according to (2) and (3), that is $p_{E P}=$ $\mathrm{I}\left(\mathrm{ETS}_{t+1}\right) v_{E P}^{\prime} \pi_{t}^{*}, p_{R T}=\mathrm{I}\left(\mathrm{RTS}_{t+1}\right) v_{R T}^{\prime} \pi_{t}^{*}, p_{E E}=1-p_{E P}, p_{R R}=1-p_{R T}$ and insert them in the transition matrix of the chain, hereby denoted by $\mathcal{T}$. 
iii. Compute the probabilities $P\left(S_{t+1}^{*} \mid \mathcal{F}_{t+1}\right)$ belonging to the vector $\pi_{t+1}^{*}$ as

$$
\pi_{t+1}^{*}=\mathcal{T}^{\prime} \pi_{t}^{*}
$$

The algorithm is initialised by assigning values to $\pi_{1}^{*}$ : if one knows that at the beginning of the sample we are in expansion, $\pi_{1}^{*} \propto v_{E}$, where $v_{E}$ is the selection vector corresponding to $\mathcal{S}_{E}$, whereas if we know that the system was in recession, $\pi_{1}^{*} \propto v_{R}$, where $v_{R}$ selects the elements of $\mathcal{S}_{R}$. Otherwise, we can learn from the first observations about the initial probability vector, and in the case these are ambiguous use a uniform prior, which amounts to set the elements of $\pi_{1}^{*}$ equal to $1 / m$.

The algorithm recursively produces $P\left(S_{t}^{*} \mid \mathcal{F}_{t}\right)$, for all $t=1, \ldots, T$, and hence, marginalising previous states $S_{t-j}, j=1,2,3,4$, the probabilities of each elementary event, $P\left(S_{t} \mid \mathcal{F}_{t}\right)$, and $P\left(\mathrm{E}_{t} \mid \mathcal{F}_{t}\right)=P\left(\mathrm{EC}_{t} \mid \mathcal{F}_{t}\right)+P\left(\mathrm{P}_{t} \mid \mathcal{F}_{t}\right), P\left(\mathrm{R}_{t} \mid \mathcal{F}_{t}\right)=P\left(\mathrm{RC}_{t} \mid \mathcal{F}_{t}\right)+P\left(\mathrm{~T}_{t} \mid \mathcal{F}_{t}\right)$, can be obtained. For instance,

$$
P\left(\mathrm{E}_{t} \mid \mathcal{F}_{t}\right)=\sum_{s_{E} \in \mathcal{S}_{E}} P\left(S_{t}^{*}=s_{E}\right)
$$

\section{A.3 Dating unobserved components}

In real applications it is usually the case that we date the business cycle on a signal extracted from a time series, rather then on the original series itself. For instance, all the series considered in this paper are seasonally adjusted (in a broad sense, including working days adjustment for the industrial production series). For deviation cycles, that are dealt with in the next section, this is usually the only available option. Therefore, we entertain unobserved components more often than we are actually aware of.

If the unobserved component, here denoted by $\varsigma_{t}$, arises from model based signal extraction techniques (for instance we aim at dating the business cycle on the seasonally adjusted series obtained from the combination of a trend-cycle component and an irregular component of known parametric form), then, apart from the obvious option of dating the sequence $\tilde{\varsigma}_{t \mid T}$, which denotes some inference (usually the expectation) on the signal conditional on the full available sample, we can score the transition probabilities using the probability of the terminating sequences, referred to the $\varsigma_{t}, \mathcal{P}_{t+1}^{(E T S)}, \mathcal{P}_{t+1}^{(R T S)}$, rather than the indicator function. The virtues of this option is that we 
are more aware of the uncertainty surrounding e.g. turning point estimation. These probabilities can be estimated via the simulation smoother of de Jong and Shephard (1995) implemented in

SsfPack. This repeatedly draws simulated samples from the posterior distribution $\tilde{\varsigma}_{t}^{(i)} \sim \psi_{t} \mid Y_{T}$, so that repeating the draws a sufficient number of times we can get Monte Carlo estimates of different aspect of the marginal and joint distribution of the terminating sequences.

Other plausible reasons for considering unobserved components are to make our dating procedure more resistant to outlier contamination and to censor variability that is not relevant to the analysis of business cycle fluctuations, such as high frequency noise. The need for the latter usually arises with reference to monthly industrial production, that even after a working days adjustment usually displays relevant high frequency components. This motivates us to employ a low pass filter, dealt with in the subsequent sections, that dampens all the fluctuations with a periodicity less than the minimum cycle duration, i.e. five quarters or 15 months.

\section{A.4 Dating the deviation cycle}

The dating algorithm of the previous section is tailored for dating classical business cycles. When we are dealing with deviation cycles (also know as growth cycles), we want to prevent that a peak is located when output is below trend levels; this is so since an expansion must have brought output above trend. Therefore, for a zero mean deviation cycle, we may want to amend the BBQ rule (and call it BBQDC) by redefining the terminating sequences as follows:

$$
\begin{aligned}
& \text { ETS }_{t}=\left\{\left(y_{t}>0\right) \cap\left(\Delta y_{t+1}<0\right) \cap\left(\Delta_{2} y_{t+2}<0\right)\right\} \\
& \text { RTS }_{t}=\left\{\left(y_{t}<0\right) \cap\left(\Delta y_{t+1}>0\right) \cap\left(\Delta_{2} y_{t+2}>0\right)\right\}
\end{aligned}
$$

The algorithm scores the cycle in real time; thus, nothing prevents that, within a period in which $y_{t}<0$, the first local minimum is flagged as a trough and that this is above the global minimum. A solution would be to run the algorithm on the reversed series, but this strategy is effective only if just two minima occur within that period. Our experience is that multiple minima are likely to occur, and thus our preferred alternative strategy works out as follows:

- Run the usual BBQ algorithm on the cumulated $y_{t}$ series, $c(y)_{t}$. The turning points detected by this procedure correspond to the crossing of the zero line. For instance a peak in $c(y)_{t}$ coincides with the latest $y_{t}>0$; all subsequent values will be below zero until a trough is 
found, which is the last point such that $y_{t}<0$. Minimum duration constraints continue to operate, but are no longer defined in terms of P-T or P-P on $y_{t}$; they relate to successive crossing of zero.

- Between two adjacent T-P turning points find the maximum of $y_{t}$ so as to locate the global peak of $y_{t}$; Between two adjacent P-T turning points find the minimum of $y_{t}$ so as to locate the global trough of $y_{t}$.

This strategy works effectively in sorting out the local minima problem and will be labelled BBQDC2.

\section{A.5 Dating monthly time series}

For monthly time series the nature of the proposed algorithms is the same. Following Bry and Boschan the minimum durations are respectively 5 months for each phase and 15 months for full cycles. This yields a 15th order MC that can be represented as a first order MC with $m=122$ states.

The terminating sequences are defined as follows:

$$
\begin{aligned}
& \text { ETS }_{t}=\left\{\bigcap_{j=1}^{5}\left(\Delta_{j} y_{t+j}<0\right)\right\} \\
& \text { RTS }_{t}=\left\{\bigcap_{j=1}^{5}\left(\Delta_{j} y_{t+j}>0\right)\right\}
\end{aligned}
$$

where $\Delta_{j}=1-L^{j}$.

\section{A.6 Depth (amplitude) restrictions}

The algorithms presented above can be readily modified to enhance depth or amplitude restrictions on the definition of expansions and recessions terminating sequences. These aim at isolating major fluctuations thereby robustifying the dating process.

Given a threshold value $c>0$, in the quarterly case we can define

$$
\begin{aligned}
& \text { ETS }_{t}=\left\{\left(\Delta y_{t+1}<-c\right) \cap\left(\Delta_{2} y_{t+2}<-c\right)\right\} \\
& \text { RTS }_{t}=\left\{\left(\Delta y_{t+1}>c\right) \cap\left(\Delta_{2} y_{t+2}>c\right)\right\}
\end{aligned}
$$

For instance, if the series we are entertaining is in logarithms $c=0.005$ could be a candidate value. 
The problem posed by amplitude constraints in dating the classical business cycle is posed by the difference in amplitude that usually characterises the two phases: expansions are longer but characterised by a lower average drift rate. This suggests that it might be the case that $c$ should vary according to the phase.

An alternative strategy uses signal extraction techniques, e.g. low-pass filters in the classical case, to isolate the most ample fluctuations. This is of course at odds with sharp turning points identification, since the probability of peaks and troughs is smeared on adjacent sample points, but certainly offers a robustification of the algorithm with respect to outliers and high frequency components.

\section{B Classical cycles, deviation cycles and growth}

It is perhaps useful to recall a few basic facts underlying the dating of the classical and deviation cycle, and illustrate them:

- Neglecting duration ties, classical recessions (i.e. P-T dynamics in the log-level $y_{t}$ ), correspond to periods of prevailing negative growth, $\Delta y_{t}<0$. In effect, negative growth is sufficient, but not necessary under Bry and Boschan dating rules. Periods of positive growth can be observed during a recession, provided that they are so short lived that they do not determine an exit from the recessionary state.

- As a matter of fact, it is immediately established that turning points in $y_{t}$ correspond to $\Delta y_{t}$ crossing zero (from above zero if the turning point is a peak, from below in the presence of a trough in $y_{t}$ ). This is strictly true under the calculus rule.

- If $y_{t}$ is $\mathrm{I}(1)$ with constant drift $\mu$, turning points in the linearly detrended series,

$$
y_{t}-\mu_{0}-\mu t
$$

correspond to $\Delta y_{t}$ crossing the mean. As a consequence, recessions correspond to periods of below average growth, $\Delta y_{t}<\mu$.

- If $y_{t}$ admits the log-additive decomposition, $y_{t}=\mu_{t}+\psi_{t}$, where $\psi_{t}$ denotes the deviation 
cycle, then growth is in turn decomposed into trend and cyclical changes:

$$
\Delta y_{t}=\Delta \mu_{t}+\Delta \psi_{t}
$$

Hence, deviation cycle recessions correspond to periods of growth below potential growth, that is $\Delta y_{t}<\Delta \mu_{t}$. This is so since, prolonged declines in $\psi_{t}$, i.e. $\Delta \psi_{t}<0$ (a deviation cycle recession), imply $\Delta y_{t}-\Delta \mu_{t}<0$. Using the same arguments, turning points correspond to $\Delta y_{t}$ crossing $\Delta \mu_{t}$. A classical recession requires that the sum of potential growth and cyclical growth is below zero, that is $\Delta \mu_{t}+\Delta \psi_{t}<0$.

- Drawing from the previous facts, classical recessions are always a subset of deviation cycle recessions, and there may be multiple classical recession episodes within a period of deviation cycle recessions.

- Current depth of recession: Beaudry and Koop (1993) proposed a measure of the depth of recession based on the deviation of current output from its historical maximum:

$$
C R D_{t}=y_{t}-\max _{j \leq t} y_{j}
$$

This measure is in the spirit of classical business cycle analysis since it is valid for series displaying systematic upward trends and takes (negative) non-zero values when an absolute decline in output occurs. However, it is a much more extreme view, as the end of the recession takes place when output catches up its historical maximum value. On the positive side it provides a measure of the amplitude of the recessionary movements. With respect to the classical definition implemented in this paper, peaks will be coincident, although $C R D_{t}$ does not impose duration ties, but a trough in our dating algorithm will tend to correspond to a trough in $C R D_{t}$, which starts moving towards zero, but will continue to be negative, signalling a recession. More local definitions. e.g. $C R D_{t}=y_{t}-\max _{k=0,1, \ldots, m} y_{t-k}$, are possible and the resulting chronology will get closer to the classical one.

- Growth rate cycle: turning points in growth rates delimit patterns of positive and negative acceleration $\left(\Delta^{2} y_{t}\right)$. ECRI produces such chronologies; according to their definition, "Growth rate cycle downturns are pronounced, pervasive and persistent declines in the growth rate 
of aggregate economic activity. The procedures used to identify peaks and troughs in the growth rate cycle are entirely analogous to those used to identify business cycle turning points, except that they are applied to the growth rates of the same time series, rather than their levels". We shall return shortly to growth rate cycles when discussing the chronology arising from the EuroCoin index.

Figure 12 illustrates all these facts with respect to the Euro area GDP series, that was preliminarily smoothed by $\mathrm{HP}(1.25)$, although we will continue to refer to it as the series, $y_{t}$, and then decomposed into a trend component and a cyclical one using respectively $\mathrm{HP}(8)$ and the residuals from it (which amounts to the HP bandpass filter $\mathrm{HP}(1.25)-\mathrm{HP}(8)$ ). The first two plots present the turning points of the classical and deviation cycle. We draw attention on the facts that the classical recessionary episode is strictly contained in the deviation one, and that the troughs are located at the same data point; although this is coincidental, the trough in $y_{t}$ cannot sequitur that in $\psi_{t}$. The third plot reproduces $\Delta y_{t}$ and potential growth, $\Delta \mu_{t}$, highlighting that deviation cycle peaks correspond to the last period of a sequence of growth above potential growth. Similarly, the deviation cycle trough corresponds to the last period of actual below potential growth. Coincidentally, this also corresponds to the last period of growth below the zero threshold. A classical peak, instead, corresponds to the last period of a sequence of positive growth rates, $\Delta y_{t}>0$.

EuroCoin, built by Altissimo et al. (2001) using dynamic factor analysis, is a composite coincident index of the Euro area growth rate cycle. As a matter of fact, it extracts the common dynamics from a large set of indicators that are made stationary by differencing.

Figure 13 presents and compares the growth rate cycle chronologies for the index and for GDP growth, using the Eurostat series from 1991.1 onwards. The turning point for the former were determined on the original monthly series and then, for comparison purposes, we aggregate the series and the dates at the quarterly frequency of observation. For GDP growth we also plot, in the third panel, the filtered series HP(1.25), removing high frequency variation, and the corresponding chronology.

ECRI states that dating the growth rate cycle requires the same rules as the classical cycle, in which case a recession implies an absolute decline in growth; when growth is stationary there is some scope also for scoring growth rate cycle as a deviation cycle, where the deviation is intended 
from the average growth rate: in such circumstance, a recession still implies a deceleration of growth, but it can be terminated only if growth is below its mean (a trough cannot be found if $\Delta y_{t}>\mu$.

There are two main noticeable facts: the first deals with the different chronology of GDP growth arising from filtering; the second is that the EuroCOIN phases agree more closely with those determined on the HP(1.25) filtered growth rates. The concordance index $I_{i j}$ between the EuroCOIN and HP(1.25) filtered GDP growth amounts to 0.8 (its expected value under the independence assumption is 0.5 ) and the concordance test statistic is 3.12. There is not much evidence for significant concordance with the raw GDP chronology $\left(I_{i j}=0.65\right.$ and the concordance statistic is 1.51 , which is not significant). The closer agreement with the $\operatorname{HP}(1.25)$ has somehow to be expected, since dynamic factor techniques smooth away high frequency movements.

\section{The Analysis of Business Cycle Information}

Harding and Pagan (2001) have stressed that, aside from the dating of their turning points, business cycles can be analysed for other information concerning their duration, amplitude and "shape". They suggest a number of measures of which we retain the three mentioned, accompanied by an expression of the probability of being in one of the two phases. The basic concept is that of the phase, of which we distinguish two - an expansion phase which lasts from the quarter following the identified trough to (and including) the peak; and a recession, which lasts from the quarter following the peak until (and including) the trough. Average phase duration is simply the number of periods (here, quarters) which an expansion, on average, contains. Amplitude is a measure of the depth of a cycle, that is the cumulative increase, during the expansion phase, of the measure of economic activity in question, whilst average amplitude is simply the average value over all expansion phases of this quantity. Dividing amplitude by duration gives a measure of steepness. Harding and Pagan (2001) suggest a "triangle analogy", for thinking about these measures, in which the upright is the amplitude, the length the duration and the hypotenuse is the expansion or recession phase. There is quite a large literature devoted to examining the exact shape of the expansion phase, in particular investigating whether the early part of the expansion of the expansion is steeper than the later part. We do not have a sufficiently large sample of 
cycles to make this an interesting exercise here. We do, however, also measure the probabilities (frequencies) of expansion and recession phase experience. Thus for a sample of complete cycles the probability of being in expansion is simply the ratio of average expansion duration to the sum of average expansion and recession durations.

Concordance statistic Given a panel of binary indicators of the state of the economy, $S_{i t}, t=$ $1, \ldots, T, i=1, \ldots, N$, available for $N$ countries, a measure of business cycle concordance between the pair of countries $i$ and $j$ is the simple matching similarity coefficient:

$$
I_{i j}=\frac{1}{T} \sum_{t=1}^{T}\left[S_{i t} S_{j t}+\left(1-S_{i t}\right)\left(1-S_{j t}\right)\right] .
$$

Let $\bar{S}_{i}=T^{-1} \sum_{t} S_{i t}$ denote the estimated probability of being in state 1 (e.g. recession); then, under the assumption that $S_{i t}$ and $S_{j t}$ are independent the estimate of the expected value of the concordance index is $2 \bar{S}_{i} \bar{S}_{j}=1-\bar{S}_{i}-\bar{S}_{j}$. Subtracting this from $I_{i j}$ gives the mean corrected concordance index (Harding and Pagan, 2001, 2002):

$$
I_{i j}^{*}=2 \frac{1}{T} \sum_{t=1}^{T}\left(S_{i t}-\bar{S}_{i}\right)\left(S_{j t}-\bar{S}_{j}\right) .
$$

The asymptotic test proposed in the paper is based on a standardised concordance index. For this purpose we need to divide $I_{i j}^{*}$ by a consistent estimate of the standard error of $I_{i j}^{*}$ under the null of independence. Now, under the null

$$
\begin{aligned}
\operatorname{Var}\left(I_{i j}\right) & =\frac{4}{T^{2}} \mathrm{E}\left[\sum_{t=1}^{T}\left(S_{i t}-E\left(S_{i t}\right)\left(S_{j t}-E\left(S_{j t}\right)\right)\right]^{2}\right. \\
& =\frac{4}{T}\left[\gamma_{i}(0) \gamma_{j}(0)+2 \sum_{\tau=1}^{T-1} \frac{T-\tau}{T} \gamma_{i}(\tau) \gamma_{j}(\tau)\right]
\end{aligned}
$$

where $\gamma_{i}(0)=\mathrm{E}\left[\left(S_{i t}-E\left(S_{i t}\right)\right)\left(S_{i, t-\tau}-E\left(S_{i t}\right)\right)\right]$.

Hence,

$$
T^{1 / 2} I_{i j}^{*} \rightarrow \mathrm{N}\left(0,4 \sigma^{2}\right), \quad \sigma^{2}=\gamma_{i}(0) \gamma_{j}(0)+2 \sum_{\tau=1}^{\infty} \gamma_{i}(\tau) \gamma_{j}(\tau)
$$

and a consistent estimate of $\sigma^{2}$ is

$$
\hat{\sigma}^{2}=\hat{\gamma}_{i}(0) \hat{\gamma}_{j}(0)+2 \sum_{\tau=1}^{l}\left(1-\frac{\tau}{T}\right) \hat{\gamma}_{i}(\tau) \hat{\gamma}_{j}(\tau),
$$

where $l$ is the truncation parameter. 


\section{Band-Pass filters}

A traditional definition of the business cycle, attributed to Burns and Mitchell (1946), considers all the fluctuations with a specified range of periodicities, namely those ranging from one and a half to eight years. Thus, if $s$ is the number of observations in a year, the fluctuations with periodicity between $1.5 s$ and $8 s$ are included.

Baxter and King (1999) argue that the ideal filter for cycle measurement must be customised to retain unaltered the amplitude of the business cycle periodic components, while removing high and low frequency components. This is known as a band-pass filter (see, e.g. Priestley, 1981), and its theoretical frequency response function takes the rectangular form: $w(\omega)=\mathrm{I}(2 \pi /(8 s) \leq \omega \leq$ $2 \pi /(1.5 s))$, where $\mathrm{I}(\cdot)$ is the indicator function. This is depicted in the second panel of figure ?? (dotted line).

Moreover, the phase displacement of the filter should always be zero, if it is to preserve the timing of peaks and troughs; the latter requirement is satisfied by a symmetric filter.

A band-pass filter is easily constructed from a low-pass filter; the latter is a filter retaining the low frequencies up to a cutoff $\omega_{0}$. Its ideal frequency response function has the form:

$$
w_{l p}(\omega)= \begin{cases}1 & \text { if }|\omega| \leq \omega_{c} \\ 0 & \text { if } \omega_{c}<|\omega| \leq \pi\end{cases}
$$

The notion of a high-pass filter is complementary as its frequency response function is $w_{h p}(\omega)=$ $1-w_{l p}(\omega)$.

The coefficients of the low-pass filter are then given by the inverse Fourier transform of $w_{l p}(\omega)$ :

$$
w_{l p}(L)=\frac{\omega_{c}}{\pi}+\sum_{j} \frac{\sin \left(\omega_{c} j\right)}{\pi j}\left(L^{j}+L^{-j}\right) .
$$

Now, given the two business cycle frequencies, $\omega_{c 1}=2 \pi /(8 s)$ and $\omega_{c 2}=2 \pi /(1.5 s)$, the band-pass filter is

$$
w_{b p}(L)=\frac{\omega_{c 2}-\omega_{c 1}}{\pi}+\sum_{j=1}^{\infty} \frac{\sin \left(\omega_{c 2} j\right)-\sin \left(\omega_{c 1} j\right)}{\pi j}\left(L^{j}+L^{-j}\right) .
$$

Thus, the ideal band-pass filter exists and is unique, but entails an infinite number of leads and lags, so in practice an approximation is required. 
Baxter and King (1999) show that the $K$-terms approximation to the ideal filter (5) that is optimal in the sense of minimising the integrated squared approximation error is simply (5) truncated at lag $K$. They propose using a three years window, i.e. $K=3 s$, as a valid rule of thumb for macroeconomic time series. They also constrain the weights to sum up to zero, so that the resulting approximation is a detrending filter: denoting the truncated filter $w_{b p, K}(L)=$ $w_{0}+\sum_{1}^{K} w_{j}\left(L^{j}+L^{-j}\right)$, the weights of the adjusted filter will be $w_{j}-w_{b p, K}(1) /(2 K+1)$.

\section{E The Hodrick-Prescott Filter}

Often the specification of the model for the trend embodies the analyst's smoothness prior on this component (see Kitagawa and Gersh, 1996). One popular example is the ad hoc trend extraction filter proposed by Hodrick and Prescott (1997), on the following grounds: "Our prior knowledge is that the growth component varies 'smoothly' over time".

HP define the estimator of the trend, $\mu_{t}$, as the minimiser of the penalised least square criterion:

$$
P L S=\sum_{t=1}^{T}\left(y_{t}-\mu_{t}\right)^{2}+\lambda \sum_{t=3}^{T}\left(\Delta^{2} \mu_{t}\right)^{2},
$$

where the first summand measures fidelity and the second roughness; $\lambda$ is the smoothness parameter governing the trade-off between them.

In vector notation, setting $y=\left(y_{1}, \ldots, y_{T}\right)^{\prime}$ and $\mu=\left(\mu_{1}, \ldots, \mu_{T}\right)^{\prime}$,

$$
P L S=(y-\mu)^{\prime}(y-\mu)+\lambda \mu^{\prime} D^{2^{\prime}} D^{2} \mu
$$

where $D$ denotes the $T \times T$ matrix corresponding to first differences, with $d_{i i}=1, d_{i, i-1}=-1$ and zero otherwise. Differentiating with respect to $\mu$, the first order conditions yield: $\tilde{\mu}=\left(I_{T}+\right.$ $\left.\lambda D^{2}\right)^{-1} y$, but the component is more efficiently computed by the Kalman filter and smoother, see Harvey (1989) and Durbin and Koopman (2001), thereby avoiding the inversion of a potentially very large matrix.

As a matter of fact, the HP filter coincides with the smoothed estimator of the trend component of the local linear trend model:

$$
\begin{array}{rlrl}
y_{t} & =\mu_{t}+\epsilon_{t}, & \epsilon_{t} & \sim \operatorname{NID}\left(0, \sigma_{\epsilon}^{2}\right), \quad t=1,2, \ldots, T, \\
\mu_{t} & =\mu_{t-1}+\beta_{t-1}+\eta_{t}, \quad \eta_{t} \sim \operatorname{NID}\left(0, \sigma_{\eta}^{2}\right), \\
\beta_{t} & \quad \beta_{t-1}+\zeta_{t}, \quad \zeta_{t} \sim \operatorname{NID}\left(0, \sigma_{\zeta}^{2}\right),
\end{array}
$$


with the restrictions $\sigma_{\eta}^{2}=0$ and $\sigma_{\epsilon}^{2} / \sigma_{\zeta}^{2}=\lambda$.

This is so since the fixed-interval smoother under Gaussianity will provide the mode of the distribution of $\mu$ conditional on $y$, whose kernel takes exactly the form (6), as $f(\mu \mid y) \propto f(y \mid \mu) f(\mu)=$ $\prod_{t} f\left(y_{t} \mid \mu_{t}\right) \prod_{t}\left(\mu_{t} \mid \mu_{t-1}, \mu_{t-2}\right)$. Notice also that the PLS problem (6) is a discrete version of the cubic spline smoothing problem.

The connection with the signal-noise ratio makes clear that the Lagrange multiplier, $\lambda$, measures the variability of the (noise) cyclical component relative to that of the trend, and regulates the smoothness of the long-term component. As $\sigma_{\eta}^{2}$ approaches zero, $\lambda$ tends to infinity, and the limiting representation of the trend is a straight line.

HP purposively select the value $\lambda=1600$ for quarterly time series; this rule has sometimes be generalised to any frequency $s$ of observations as $\lambda=100 s^{2}$. The value 100 is used by the European Commission for measuring potential output on yearly series which are used in the calculation of cyclically adjusted budget balances. The HP detrended or cyclical component is the smoothed estimate of the irregular component in (7) and, although the maintained representation for the deviations from the trend is a purely irregular component, the filter has been one of the most widely employed tools in macroeconomics to extract measures of the business cycle.

\section{E.1 Designing the HP filter as a Lowpass filter}

The theoretical properties of the HP filter are better understood assuming the availability of a doubly infinite sample, $y_{t+j}, j=-\infty, \ldots, \infty$. In such setting, the Wiener-Kolmogorov filter (see Whittle, 1963) provides the minimum mean square linear estimator (MMSLE) of the trend:

$$
\tilde{\mu}_{t \mid \infty}=w_{H P}(L) y_{t},
$$

where

$$
w_{H P}(L)=\frac{\sigma_{\zeta}^{2}}{\sigma_{\zeta}^{2}+|1-L|^{2} \sigma_{\epsilon}^{2}}=\frac{1}{1+\lambda|1-L|^{4}}
$$

and we have written $|1-L|^{2}=(1-L)\left(1-L^{-1}\right)$. Replacing $L=1$ in the above expression, it can be seen that the weights sum up to one.

The frequency response function of the filter is:

$$
w_{H P}\left(e^{-\imath \omega}\right)=\frac{1}{1+4 \lambda(1-\cos \omega)^{2}}
$$


notice that this is 1 at the zero frequency and decreases monotonically to zero as $\omega$ approaches $\pi$. Its behaviour enforces the interpretation of (8) as a low-pass filter, and the corresponding detrending filter, $1-w_{H P}(L)$, is the high-pass filter derived from it.

The implicit cut-off frequency is the value $\omega_{c}$ corresponding to a gain $\left|w_{H P}\left(e^{-\imath \omega}\right)\right|=1 / 2$. This satisfies the equation

$$
\lambda=\left[4\left(1-\cos \omega_{c}\right)^{2}\right]^{-1}
$$

for a given smoothness parameter (9) can be solved with respect to the cut-off frequency $\omega_{c}$, giving $\omega_{c}=\arccos \left(1-0.5 \lambda^{-1 / 2}\right)$. For instance, setting $\lambda=1600$ we get that the HP filter for quarterly data is a low-pass filter with $\omega_{c}=0.158$ corresponding to a period of 39.69 e.g. 10 years, which is strictly outside the range proposed by Baxter and King (1999). Similar arguments enable to conclude that the value $\lambda=100$ defines a low-pass filter retaining fluctuations with period larger than 20 years.

Solving equation (9) for $\lambda$, given a specific cutoff $\omega_{c}$, provides a way of designing the filter as a low-pass filter. As a first application, let us express the the period as a function of $s$, e.g. $p=\bar{p} s$, where $\bar{p}$ is a fixed period in years, replacing into $\omega_{c}=2 \pi / p=2 \pi /(\bar{p} s),(9)$ shows that there are no simple rules of the kind $\lambda \propto s^{2}$ for different frequencies of observations, if one wants to keep the period in years $\bar{p}$ constant; see Kaiser and Maravall (2001).

As a second application, we can use the result to design a band-pass filter for business cycle extraction as the difference of two HP detrending filters, the first for $\omega_{c}=2 \pi /(1.5 s)$ and the second for $\omega_{c}=2 \pi /(8 s)$ : for quarterly data it is easy to show that the cycle will result from the difference of two trend estimates, the first with $\lambda=1$ and the second with $\lambda=677.13$.

\section{F Model-based filters}

In a recent paper, Harvey and Trimbur (2002) have proposed a general class of model based filters for extracting trend and cycles in macroeconomic time series, showing that the design of lowpass and band-pass filters can be considered as a signal extraction problem in an unobserved components framework. 
They consider the class of $m$-th order stochastic trend:

$$
\begin{aligned}
& \mu_{1 t}=\mu_{1, t-1}+\zeta_{t} \\
& \mu_{i t}=\mu_{i, t-1}+\mu_{i-1, t}, \quad i=2, \ldots, m
\end{aligned}
$$

This is the recursive definition of an $m$-fold integrated random walk, such that $\Delta^{m} \mu_{m t}=\zeta_{t}$. When the observational model is $y_{t}=\mu_{m t}+\varepsilon_{t}$, the Wiener-Kolmogorov trend extraction filter is a low-pass filter belonging to the Butterworth family:

$$
w_{\mu, m}(L)=\frac{1}{1+q^{-1}|1-L|^{2 m}}, \quad q=\sigma_{\zeta}^{2} / \sigma_{\epsilon}^{2}
$$

Notice that $m=2$ gives exactly the HP filter; $m=1$ produces the signal extraction filter for local level model, producing an exponentially two-sided moving average of the available observations. As $m$ increases, the filter is a close approximation to the ideal low-pass filter.

Similarly, an $n$-th order stochastic cycle is defined as:

$$
\begin{gathered}
{\left[\begin{array}{l}
\psi_{1 t} \\
\psi_{1 t}^{*}
\end{array}\right]=\rho\left[\begin{array}{cc}
\cos \lambda_{c} & \sin \lambda_{c} \\
-\sin \lambda_{c} & \cos \lambda_{c}
\end{array}\right]\left[\begin{array}{l}
\psi_{1, t-1} \\
\psi_{1, t-1}^{*}
\end{array}\right]+\left[\begin{array}{c}
\kappa_{t} \\
0
\end{array}\right],} \\
{\left[\begin{array}{l}
\psi_{i t} \\
\psi_{i t}^{*}
\end{array}\right]=\rho\left[\begin{array}{cc}
\cos \lambda_{c} & \sin \lambda_{c} \\
-\sin \lambda_{c} & \cos \lambda_{c}
\end{array}\right]\left[\begin{array}{l}
\psi_{i, t-1} \\
\psi_{i, t-1}^{*}
\end{array}\right]+\left[\begin{array}{c}
\psi_{i-1, t} \\
0
\end{array}\right],}
\end{gathered}
$$

The univariate representation for such a process is:

$$
\psi_{n t}=\left[\frac{1-\rho \cos \lambda_{c} L}{1-2 \rho \cos \lambda_{c} L+\rho^{2} L^{2}}\right]^{n} \kappa_{t}
$$

and Harvey and Trimbur show that if the model is

$$
y_{t}=\mu_{m t}+\psi_{n t}+\epsilon_{t}
$$

the optimal estimators of the trend and the cycle are the generalised low and band pass Butterworth filters of order $(m, n)$. In finite sample they are produced by the Kalman filter and smoother. 
Table 9: Description of the Markov chain generated by the quarterly dating rules.

\begin{tabular}{|c|c|c|c|c|c|c|c|c|c|}
\hline States & $S_{t}^{*}=$ & $S_{t-4}$, & $t-3, S$ & $-2, S_{t-}$ & $\left.S_{t}\right\}$ & & States $S_{t+1}^{*}$ & at can & isited \\
\hline$S_{t}^{*}$ & $S_{t-4}$ & $S_{t-3}$ & $S_{t-2}$ & $S_{t-1}$ & $S_{t}$ & $S_{t+1}^{*}$ & Trans. Prob. & $S_{t+1}^{*}$ & Trans. Prob. \\
\hline$S_{1}^{*}$ & $P$ & $\mathrm{RC}$ & $\mathrm{RC}$ & $\mathrm{RC}$ & $\mathrm{RC}$ & $S_{17}^{*}$ & $p_{R R}$ & $S_{18}^{*}$ & $p_{R T}$ \\
\hline$S_{2}^{*}$ & $P$ & $\mathrm{RC}$ & $\mathrm{RC}$ & $\mathrm{RC}$ & $\mathrm{T}$ & $S_{19}^{*}$ & 1 & & \\
\hline$S_{3}^{*}$ & $P$ & $\mathrm{RC}$ & $\mathrm{RC}$ & $\mathrm{T}$ & EC & $S_{20}^{*}$ & $p_{E E}$ & $S_{21}^{*}$ & $p_{E P}$ \\
\hline$S_{4}^{*}$ & $P$ & $\mathrm{RC}$ & $\mathrm{T}$ & EC & EC & $S_{22}^{*}$ & $p_{E E}$ & $S_{23}^{*}$ & $p_{E P}$ \\
\hline$S_{5}^{*}$ & $\mathrm{~T}$ & EC & EC & EC & EC & $S_{9}^{*}$ & $p_{E E}$ & $S_{10}^{*}$ & $p_{E P}$ \\
\hline$S_{6}^{*}$ & $\mathrm{~T}$ & EC & EC & EC & $P$ & $S_{11}^{*}$ & 1 & & \\
\hline$S_{7}^{*}$ & $\mathrm{~T}$ & EC & EC & $\mathrm{P}$ & $\mathrm{RC}$ & $S_{12}^{*}$ & $p_{R R}$ & $S_{13}^{*}$ & $p_{R T}$ \\
\hline$S_{8}^{*}$ & $\mathrm{~T}$ & EC & $\mathrm{P}$ & $\mathrm{RC}$ & $\mathrm{RC}$ & $S_{14}^{*}$ & $p_{R R}$ & $S_{15}^{*}$ & $p_{R T}$ \\
\hline$S_{9}^{*}$ & EC & EC & EC & EC & EC & $S_{9}^{*}$ & $p_{E E}$ & $S_{10}^{*}$ & $p_{E P}$ \\
\hline$S_{10}^{*}$ & EC & EC & EC & EC & $\mathrm{P}$ & $S_{11}^{*}$ & 1 & & \\
\hline$S_{11}^{*}$ & EC & EC & EC & $\mathrm{P}$ & $\mathrm{RC}$ & $S_{12}^{*}$ & $p_{R R}$ & $S_{13}^{*}$ & $p_{R T}$ \\
\hline$S_{12}^{*}$ & EC & EC & $\mathrm{P}$ & $\mathrm{RC}$ & $\mathrm{RC}$ & $S_{14}^{*}$ & $p_{R R}$ & $S_{15}^{*}$ & $p_{R T}$ \\
\hline$S_{13}^{*}$ & EC & EC & P & $\mathrm{RC}$ & $\mathrm{T}$ & $S_{16}^{*}$ & 1 & & \\
\hline$S_{14}^{*}$ & EC & $P$ & $\mathrm{RC}$ & $\mathrm{RC}$ & $\mathrm{RC}$ & $S_{1}^{*}$ & $p_{R R}$ & $S_{2}^{*}$ & $p_{R T}$ \\
\hline$S_{15}^{*}$ & EC & P & $\mathrm{RC}$ & $\mathrm{RC}$ & $\mathrm{T}$ & $S_{3}^{*}$ & 1 & & \\
\hline$S_{16}^{*}$ & EC & $P$ & $\mathrm{RC}$ & $\mathrm{T}$ & EC & $S_{4}^{*}$ & 1 & & \\
\hline$S_{17}^{*}$ & $\mathrm{RC}$ & $\mathrm{RC}$ & $\mathrm{RC}$ & $\mathrm{RC}$ & $\mathrm{RC}$ & $S_{17}^{*}$ & $p_{R R}$ & $S_{18}^{*}$ & $p_{R T}$ \\
\hline$S_{18}^{*}$ & $\mathrm{RC}$ & $\mathrm{RC}$ & $\mathrm{RC}$ & $\mathrm{RC}$ & $\mathrm{T}$ & $S_{19}^{*}$ & 1 & & \\
\hline$S_{19}^{*}$ & $\mathrm{RC}$ & $\mathrm{RC}$ & $\mathrm{RC}$ & $\mathrm{T}$ & EC & $S_{20}^{*}$ & $p_{E E}$ & $S_{21}^{*}$ & $p_{E P}$ \\
\hline$S_{20}^{*}$ & $\mathrm{RC}$ & $\mathrm{RC}$ & $\mathrm{T}$ & EC & EC & $S_{22}^{*}$ & $p_{E E}$ & $S_{23}^{*}$ & $p_{E P}$ \\
\hline$S_{21}^{*}$ & $\mathrm{RC}$ & $\mathrm{RC}$ & $\mathrm{T}$ & EC & $\mathrm{P}$ & $S_{24}^{*}$ & 1 & & \\
\hline$S_{22}^{*}$ & RC & $\mathrm{T}$ & EC & EC & EC & $S_{5}^{*}$ & $p_{E E}$ & $S_{6}^{*}$ & $p_{E P}$ \\
\hline$S_{23}^{*}$ & $\mathrm{RC}$ & $\mathrm{T}$ & EC & EC & $P$ & $S_{7}^{*}$ & 1 & & \\
\hline$S_{24}^{*}$ & $\mathrm{RC}$ & $\mathrm{T}$ & EC & $\mathrm{P}$ & $\mathrm{RC}$ & $S_{8}^{*}$ & 1 & & \\
\hline
\end{tabular}



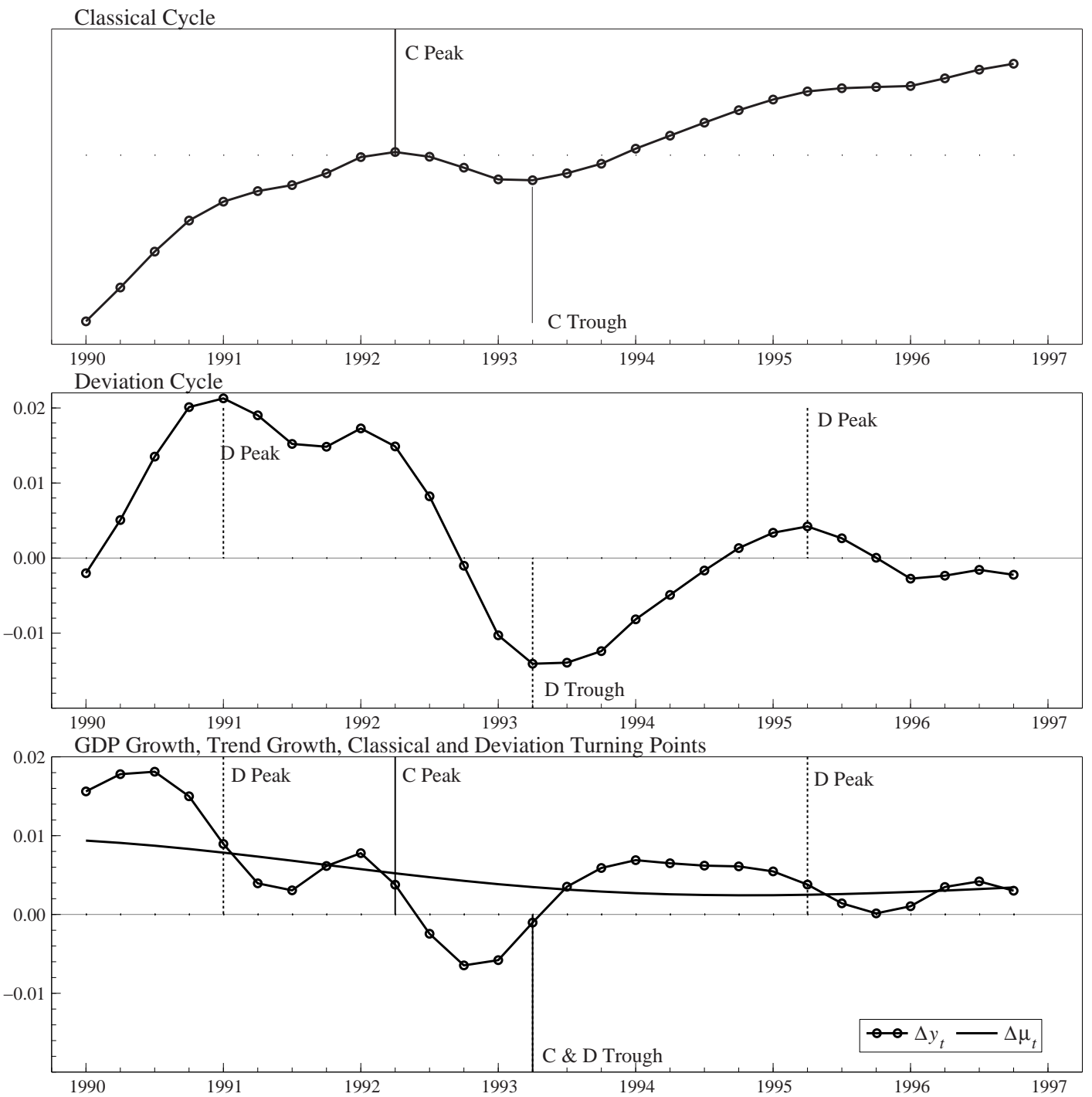

Figure 12: Classical and deviation cycles turning points and their relation with growth. 

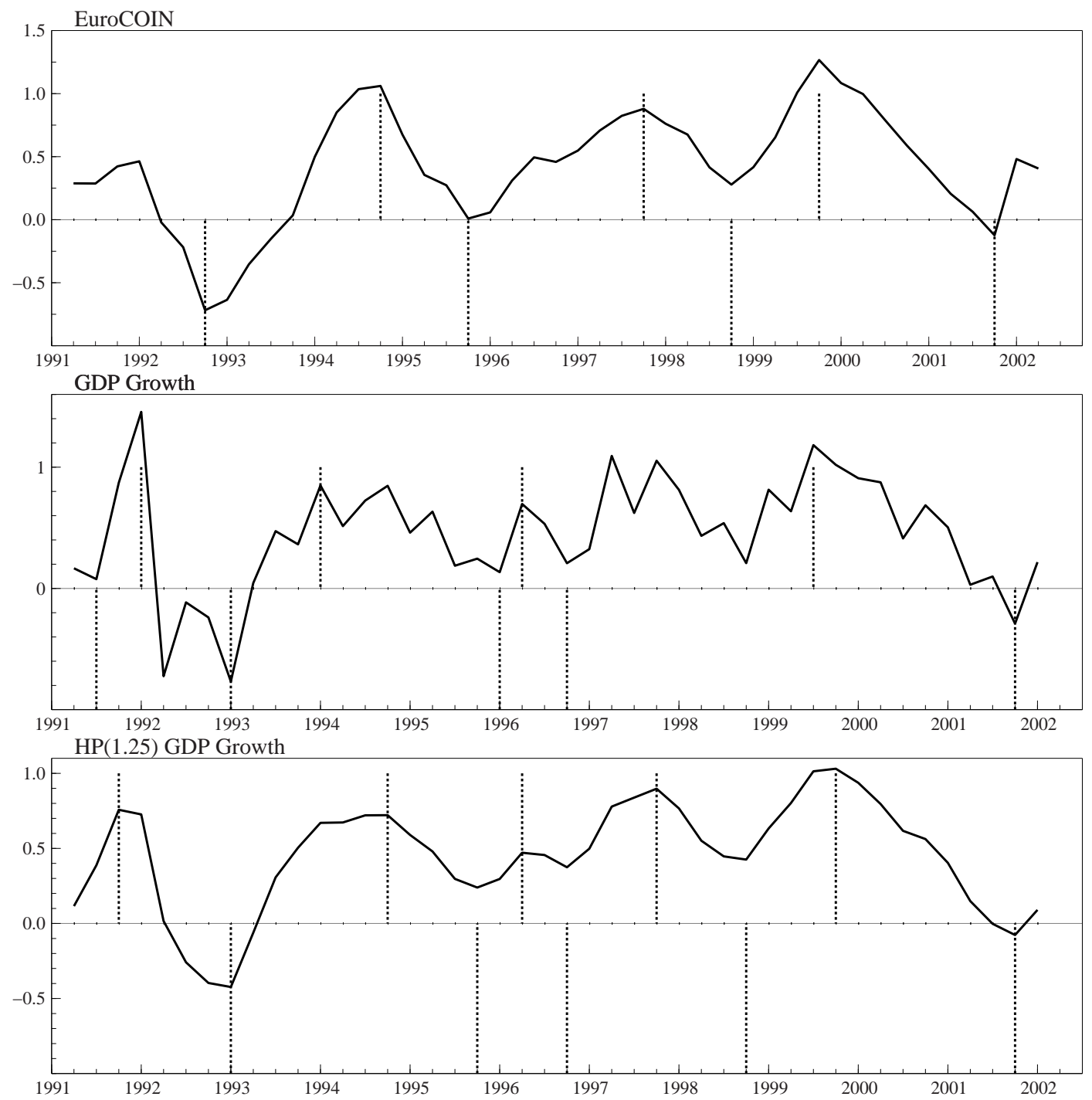

Figure 13: Growth rate cycles for EuroCOIN index and raw and filtered GDP growth. 